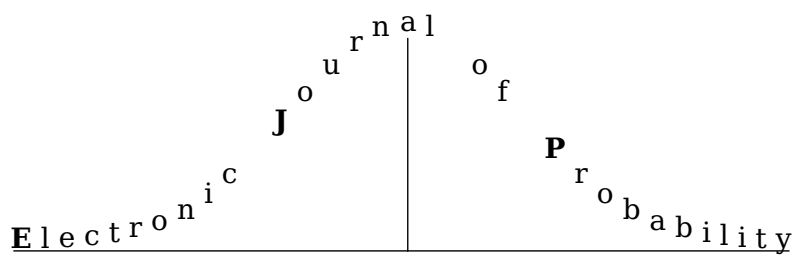

Electron. J. Probab. 26 (2021), article no. 92, 1-26.

ISSN: 1083-6489 https://doi.org/10.1214/21-EJP661

\title{
The invariant measure of PushASEP with a wall and point-to-line last passage percolation*
}

\author{
Will FitzGerald ${ }^{\dagger}$
}

\begin{abstract}
We consider an interacting particle system on the lattice involving pushing and blocking interactions, called PushASEP, in the presence of a wall at the origin. We show that the invariant measure of this system is equal in distribution to a vector of point-to-line last passage percolation times in a random geometrically distributed environment. The largest co-ordinates in both of these vectors are equal in distribution to the all-time supremum of a non-colliding random walk.
\end{abstract}

Keywords: interacting particle systems; non-colliding random walks; point-to-line last passage percolation; symplectic Schur functions.

MSC2020 subject classifications: 60K35; 60C05; 60J45.

Submitted to EJP on October 29, 2020, final version accepted on June 7, 2021.

\section{Introduction}

The last two decades have seen remarkable progress in the study of random interface growth, interacting particle systems and random polymers within the Kardar-ParisiZhang (KPZ) universality class through the identification of deep connections between probability, combinatorics, symmetric functions, queueing theory, random matrices and quantum integrable systems. The greatest progress has been made with narrow-wedge initial data (for example see $[1,3,7,12,18,26,27,31]$ ) and there are substantial differences in the case of flat initial data, see [2, 6, 10, 15, 24, 29].

The purpose of this paper is to prove multi-dimensional identities in law between different models in the KPZ universality class with flat initial data. These are closely related to identities involving reflected Brownian motions and point-to-line last passage percolation with exponential data proved recently in [16]. The results of this paper, together with [16], suggests the possibility that there may be more identities of this form and deeper algebraic reasons for why they hold.

\footnotetext{
*I am grateful for the financial support of the Royal Society Enhancement Award 'Log-correlated Gaussian fields and symmetry classes in random matrix theory RGF|EA|181085.'

${ }^{\dagger}$ University of Sussex, United Kingdom. E-mail: w. fitzgerald@sussex.ac.uk
} 
On the one hand, these identities involve an interacting particle system called PushASEP (introduced in [8]) in the presence of an additional wall at the origin. This is a continuous-time Markov chain $\left(Y_{1}(t), \ldots, Y_{n}(t)\right)_{t \geq 0}$ taking values in $W_{\geq 0}^{n}=\left\{\left(y_{1}, \ldots, y_{n}\right)\right.$ : $0 \leq y_{1} \ldots \leq y_{n}$ and $\left.y_{i} \in \mathbb{Z}\right\}$ and with the following evolution depending on $2 n$ independent exponential clocks. Throughout we refer to the $i$-th co-ordinate as the $i$-th particle. At rate $v_{i}$, the right-clock of the $i$-th particle rings and the $i$-th particle jumps to the right. All particles which have (before the jump of the $i$-th particle) a position equal to the $i$-th particle position and an index greater than or equal to $i$ are pushed by one step to the right. At rate $v_{i}^{-1}$ the left-clock of the $i$-th particle rings and if the $i$-th particle has a position strictly larger than both the $(i-1)$-th particle and zero then the $i$-th particle jumps by one step to the left; if not this jump is suppressed. In summary, particles push particles with higher indices and are blocked by particles with lower indices and a wall at the origin. Our focus will be on the case $0<v_{1}, \ldots, v_{n}<1$ when this process has a stationary distribution.

A second viewpoint is to relate the top particle in PushASEP with a wall to the top particle in an ordered (or non-colliding process), see Proposition 2.1 and related statements in $[3,4,26,32]$. Let $\left(Z_{1}^{\left(v_{n}\right)}(t), \ldots, Z_{n}^{\left(v_{1}\right)}(t)\right)_{t \geq 0}$ be a multi-dimensional continuoustime random walk started from $(0, \ldots, 0)$ where $Z_{i}^{\left(v_{n-i+1}\right)}$ jumps to the right with rate $v_{n-i+1}$ and to the left with rate $v_{n-i+1}^{-1}$. We construct from this an ordered process $\left(Z_{1}^{\dagger}(t), \ldots, Z_{n}^{\dagger}(t)\right)_{t \geq 0}$ by a Doob $h$-transform, see Section 2 . In the case $0<v_{n}<\ldots<v_{1}$, this is given by conditioning $\left(Z_{1}^{\left(v_{n}\right)}, \ldots, Z_{n}^{\left(v_{1}\right)}\right)$ on the event of positive probability that $Z_{1}^{\left(v_{n}\right)} \leq \ldots \leq Z_{n}^{\left(v_{1}\right)}$.

The other side of the identities we prove, involve point-to-line last passage percolation times. Let $\Pi_{n}^{\text {flat }}(k, l)$ denote the set of all directed (up and right) nearest neighbour paths from the point $(k, l)$ to the line $\{(i, j): i+j=n+1\}$ and let

$$
G(k, l)=\max _{\pi \in \Pi_{n}^{\mathrm{fat}}(k, l)} \sum_{(i, j) \in \pi} g_{i j}
$$

where $g_{i j}$ are an independent collection of geometric random variables with parameter $1-v_{i} v_{n-j+1}$ indexed by $\left\{(i, j): i, j \in \mathbb{Z}_{\geq 1}\right.$ and $\left.i+j \leq n+1\right\}$ and with $0<v_{i}<1$ for each $i=1, \ldots, n$. The geometric random variables are defined as $P\left(g_{i j}=k\right)=$ $\left(1-v_{i} v_{n-j+1}\right)\left(v_{i} v_{n-j+1}\right)^{k}$ for all $k \geq 0$.

Theorem 1.1. Let $n \geq 1$ and suppose $0<v_{1}, \ldots, v_{n}<1$ and let $Y_{n}^{*}$ be distributed according to the top particle of PushASEP with a wall in its invariant measure, let $Z_{n}^{\dagger}$ be the top particle in the ordered random walk above and $G(1,1)$ be the point-to-line last passage percolation time defined by (1.1). Then

$$
Y_{n}^{*} \stackrel{d}{=} \sup _{t \geq 0} Z_{n}^{\dagger}(t) \stackrel{d}{=} G(1,1)
$$

The first identity in Theorem 1.1 follows from two representations for $Y_{n}^{*}$ and $\sup _{t \geq 0} Z_{n}^{\dagger}(t)$ as point-to-line last passage percolation times in a random environment constructed from Poisson point processes. The equality in law then follows from a time reversal argument.

The main content of Theorem 1.1 is that either of these random variables is equal in distribution to a point-to-line last passage percolation time. We provide two proofs; the first is more direct while the second proves Theorem 1.1 as a corollary of our main result in Theorem 2. The first method is to calculate the distribution function of $\sup _{t \geq 0} Z_{n}^{\dagger}(t)$ by relating the problem to conditioning a multi-dimensional random walk to stay in a Weyl chamber of type $\mathrm{C}$ given that it remains in a Weyl chamber of type A. This gives the distribution function of $\sup _{t \geq 0} Z_{n}^{\dagger}(t)$ as proportional to a symplectic Schur function 
divided by a Schur function. This can be identified as a known expression for the distribution function of point-to-line last passage percolation in a geometric environment from [5]. This proof of Theorem 1.1 is given in Section 2.

The second method of proof is to view Theorem 1.1 as an equality of the marginal distributions of the largest co-ordinates in a multi-dimensional identity in law relating the whole invariant measure of PushASEP with a wall to a vector of point-to-line last passage percolation times. This leads to our main result.

Theorem 1.2. Let $n \geq 1$ and suppose $0<v_{1}, \ldots, v_{n}<1$. Let $\left(Y_{1}^{*}, \ldots, Y_{n}^{*}\right)$ be distributed according to the invariant measure of PushASEP with a wall and let $(G(1, n), \ldots, G(1,1))$ be a vector of point-to-line last passage percolation times defined in (1.1). Then

$$
\left(Y_{1}^{*}, \ldots, Y_{n}^{*}\right) \stackrel{d}{=}(G(1, n), \ldots, G(1,1)) .
$$

We give two proofs of Theorem 1.2; one motivation is that each proof establishes different intermediate statements which we believe will be useful in their own right. In the first proof, we prove in Section 3 a formula for the transition probability of PushASEP with a wall, following the method of [8]. From this we obtain an expression for the probability mass function of $\left(Y_{1}^{*}, \ldots, Y_{n}^{*}\right)$ in Proposition 3.3. In Section 4, we use an interpretation of last passage percolation as a discrete-time Markov chain, with a sequential update rule for particle positions, which has explicit determinantal transition probabilities given in [14]. In order to find the distribution of a vector of point-to-line last passage percolation times, we use the update rule of this discrete-time Markov chain while adding in a new particle at the origin after each time step. In such a way we can find an explicit probability mass function for $(G(1, n), \ldots, G(1,1))$ which agrees with $\left(Y_{1}^{*}, \ldots, Y_{n}^{*}\right)$ and gives our first proof of Theorem 1.2.

The second proof of Theorem 1.2 is to obtain this multi-dimensional equality in law as a marginal equality of a larger identity in law. We give this proof in Section 5. In particular, we construct a multi-dimensional Markov process involving pushing and blocking interaction which has (i) an invariant measure given by $\{G(i, j): i+j \leq n+1\}$ and (ii) a certain marginal given by PushASEP with a wall. Moreover, the process we construct has the same distribution when run forwards and backwards in time up to a relabelling of the co-ordinates. This is an example of dynamical reversibility. (Dynamical reversibility is more general than the above statement and we refer to Kelly [20] for the definition in full generality and applications to queueing networks.) Dynamical reversibility leads to a convenient way of finding an invariant measure and can be used to deduce further properties of PushASEP with a wall. In particular, when started in stationarity the top particle of PushASEP with a wall evolves as a non-Markovian process with the same distribution when run forwards and backwards in time. This is a property shared by the Airy ${ }_{1}$ process and it is natural to expect that the top particle in PushASEP with a wall run in stationarity converges (up to a rescaling) to the Airy ${ }_{1}$ process.

We end the introduction by comparing with the results on PushASEP in Borodin and Ferrari [8]. When started from a step or periodic initial condition [8] prove that the associated height function converges (up to a rescaling) to the Airy or Airy $_{1}$ process respectively (see also the seminal work [10, 29]). The choice of a periodic initial condition thus gives one way of accessing the KPZ universality class started from a flat interface. In this paper we instead impose a wall at the origin and consider the invariant measure of PushASEP with a wall. This makes a substantial difference to the analysis and unveils different connections within the KPZ universality class with flat initial data. 


\section{Proof of Theorem 1.1}

\subsection{The all-time supremum of a non-colliding process}

We start by defining Schur and symplectic Schur functions. It will be sufficient for our purposes to define them according to their Weyl character formulas and we only remark that they can also be defined as a sum over weighted Gelfand Tsetlin patterns and have a representation theoretic significance, see [17]. Let $W^{n}=\left\{\left(x_{1}, \ldots, x_{n}\right) \in \mathbb{Z}^{n}\right.$ : $\left.x_{1} \leq \ldots \leq x_{n}\right\}, W_{\geq 0}^{n}=\left\{\left(x_{1}, \ldots, x_{n}\right) \in \mathbb{Z}^{n}: 0 \leq x_{1} \leq \ldots \leq x_{n}\right\}$ and $W_{\leq 0}^{n}=\left\{\left(x_{1}, \ldots, x_{n}\right) \in\right.$ $\left.\mathbb{Z}^{n}: x_{1} \leq \ldots \leq x_{n} \leq 0\right\}$. For $x \in W^{n}$ we define the Schur function $S_{x}^{\leq}: \mathbb{R}^{n} \rightarrow \mathbb{R}$ by

$$
S_{x}(v)=\frac{\operatorname{det}\left(v_{i}^{x_{j}+j-1}\right)_{i, j=1}^{n}}{\operatorname{det}\left(v_{i}^{j-1}\right)_{i, j=1}^{n}}
$$

and for $x \in W_{\geq 0}^{n}$ we define the symplectic Schur function $\mathrm{Sp}_{x}: \mathbb{R}_{>0}^{n} \rightarrow \mathbb{R}$ by

$$
\operatorname{Sp}_{x}(v)=\frac{\operatorname{det}\left(v_{i}^{x_{j}+j}-v_{i}^{-\left(x_{j}+j\right)}\right)_{i, j=1}^{n}}{\operatorname{det}\left(v_{i}^{j}-v_{i}^{-j}\right)_{i, j=1}^{n}} .
$$

Let $\left(Z_{1}^{\left(v_{n}\right)}(t), \ldots, Z_{n}^{\left(v_{1}\right)}(t)\right)_{t \geq 0}$ denote a multi-dimensional continuous-time random walk started from $\left(x_{1}, \ldots, x_{n}\right)$ where each component is independent and $Z_{i}^{\left(v_{n-i+1}\right)}$ jumps to the right at rate $v_{n-i+1}$ and to the left with rate $v_{n-i+1}^{-1}$. We define an ordered random walk $\left(Z_{1}^{\dagger}(t), \ldots, Z_{n}^{\dagger}(t)\right)_{t \geq 0}$ started from $x \in W^{n}$ as having a $Q$-matrix (generator) given by a Doob $h$-transform: for $x \in W^{n}$ and $i=1, \ldots, n$, with off-diagonal entries

$$
Q_{Z^{\dagger}}\left(x, x \pm e_{i}\right)=\frac{S_{x \pm e_{i}}(v)}{S_{x}(v)} 1_{\left\{x \pm e_{i} \in W^{n}\right\}} .
$$

The diagonal entries are given for $x \in W^{n}$ by $Q_{Z^{\dagger}}(x, x)=-\sum_{i=1}^{n}\left(v_{i}^{-1}+v_{i}\right)$. The fact that this is a conservative $Q$-matrix is part of Proposition 2.1. This is a version of $\left(Z_{1}^{\left(v_{n}\right)}(t), \ldots, Z_{n}^{\left(v_{1}\right)}(t)\right)_{t \geq 0}$ with components conditioned to remain ordered as $Z_{1} \leq \ldots \leq$ $Z_{n}$. It is related to a non-colliding random walk with components conditioned to remain strictly ordered by a co-ordinate change; for more information on non-colliding random walks we refer to [21, 22, 25].

Define $h_{A}: W^{n} \rightarrow \mathbb{R}$ by $h_{A}(x)=\prod_{i=1}^{n} v_{n-i+1}^{-x_{i}} S_{x}(v)$ and define $h_{C}: W_{\leq 0}^{n} \rightarrow \mathbb{R}$ by $h_{C}\left(x_{1}, \ldots, x_{n}\right)=\prod_{i=1}^{n} v_{n-i+1}^{-x_{i}} \operatorname{Sp}_{\left(-x_{n}, \ldots,-x_{1}\right)}(v)$.

Proposition 2.1. (i) $Q_{Z^{\dagger}}$ is a conservative $Q$-matrix. Equivalently, $h_{A}$ is harmonic for $\left(Z_{1}^{\left(v_{n}\right)}(t), \ldots, Z_{n}^{\left(v_{1}\right)}(t)\right)_{t \geq 0}$ killed when it leaves $W^{n}$.

(ii) We have that

$$
\left(Z_{n}^{\dagger}(t)\right)_{t \geq 0} \stackrel{d}{=}\left(\sup _{0=t_{0} \leq t_{1} \leq \ldots \leq t_{n}=t} \sum_{i=1}^{n}\left(Z_{i}^{\left(v_{n-i+1}\right)}\left(t_{i}\right)-Z_{i}^{\left(v_{n-i+1}\right)}\left(t_{i-1}\right)\right)\right)_{t \geq 0} .
$$

This is a consequence of Theorem 5.10 in [4] and is proved by multidimensional versions of Pitman's transformation. It is also closely related to the analysis in [8]. In the case that only rightward jumps in $Z_{i}$ are present, this corresponds to a construction of a process on a Gelfand-Tsetlin patten with pushing and blocking interactions [33]. The statement above can also be proved as a consequence of push-block dynamics by minor modifications of the proof of Theorem 2.1 in [33] and we describe these modifications in Section 6 . The construction of a corresponding process on a symplectic Gelfand Tsetlin pattern in [33] leads to the following. 
Lemma 2.2 (Theorem 2.3 of [33]). $h_{C}$ is harmonic for $\left(Z_{1}^{\left(v_{n}\right)}, \ldots, Z_{n}^{\left(v_{1}\right)}\right)$ killed when it leaves $W_{\leq 0}^{n}$.

This is a reflection through the origin of the result in [33] which considers a process killed when it leaves $W_{\geq 0}^{n}$.

Proposition 2.3 (Corollary 7.7 of [23]). Suppose $0<v_{n}<\ldots<v_{1}<1$.

(i) Let $T_{A}=\inf \left\{t \geq 0:\left(Z_{1}^{\left(v_{n}\right)}(t), \ldots, Z_{n}^{\left(v_{1}\right)}(t)\right) \notin W^{n}\right\}$. Then for $x \in W^{n}$, we have $P_{x}\left(T_{A}=\infty\right)=\kappa_{A} h_{A}(x)$ where

$$
\kappa_{A}=\prod_{i<j}\left(v_{i}-v_{j}\right) \prod_{j=1}^{n-1} v_{j}^{-(n-j)} .
$$

(ii) Let $T_{C}=\inf \left\{t \geq 0:\left(Z_{1}^{\left(v_{n}\right)}(t), \ldots, Z_{n}^{\left(v_{1}\right)}(t)\right) \notin W_{\leq 0}^{n}\right\}$. Then for $x \in W_{\leq 0}^{n}$, we have $P_{x}\left(T_{C}=\infty\right)=\kappa_{C} h_{C}(x)$ where

$$
\kappa_{C}=\prod_{1 \leq i \leq j \leq n}\left(1-v_{i} v_{j}\right) \prod_{i<j}\left(v_{i}-v_{j}\right) \prod_{j=1}^{n-1} v_{j}^{-(n-j)}
$$

The probability that a random walk remains within a Weyl chamber for all time is considered in a general setting in [23]. In our setting, we give a direct proof using Proposition 2.1 and Lemma 2.2.

Proof. Proposition 2.1 and Lemma 2.2 show that $h_{A}$ and $h_{C}$ are harmonic functions for $\left(Z^{\left(v_{n}\right)}(t), \ldots, Z^{\left(v_{1}\right)}\right)$ killed when it leaves $W^{n}$ and $W_{\leq 0}^{n}$ respectively.

We now check that $\kappa_{A} h_{A}$ and $\kappa_{C} h_{C}$ have the correct boundary behaviour. Let $|x|=\sum_{i=1}^{d} x_{i}$ for $x \in \mathbb{R}^{d}$ and define $\partial W^{n}=\left\{x \notin W^{n}: \exists x^{\prime} \in W^{n}\right.$ with $\left.\left|x-x^{\prime}\right|=1\right\}$ and $\partial W_{\leq 0}^{n}=\left\{x \notin W_{\leq 0}^{n}: \exists x^{\prime} \in W_{\leq 0}^{n}\right.$ with $\left.\left|x-x^{\prime}\right|=1\right\}$. Then we can observe from (2.1) that $S_{x}(v)=0$ for all $x \in \partial W^{n}$ because two columns in the determinant in the numerator of (2.1) coincide if $x_{i}=x_{i+1}+1$ for some $i=1, \ldots, n-1$. In a similar manner, $h_{C}\left(x_{1}, \ldots, x_{n}\right)=\prod_{i=1}^{n} v_{n-i+1}^{-x_{i}} \operatorname{Sp}_{\left(-x_{n}, \ldots,-x_{1}\right)}(v)=0$ for all $x \in \partial W_{\leq 0}^{n}$ due to the above observation and that $h_{C}(x)=0$ when $x_{n}=1$.

We now consider the behaviour at infinity. For $h_{A}$, it is easy to see from the Weyl character formula (2.1) that

$$
\kappa_{A} \lim _{x_{i}-x_{i+1} \rightarrow-\infty} \prod_{i=1}^{n} v_{n-i+1}^{-x_{i}} S_{x}(v)=\kappa_{A} \frac{\prod_{j=1}^{n-1} v_{j}^{n-j}}{\prod_{i<j}\left(v_{i}-v_{j}\right)}=1
$$

where we use the limit above to mean $x_{1}, \ldots, x_{n} \rightarrow-\infty$ and $x_{i}-x_{i+1} \rightarrow-\infty$ for each $i=1, \ldots, n-1$. For the symplectic Schur function we find

$$
\lim _{x_{i}-x_{i+1} \rightarrow-\infty} \prod_{i=1}^{n} v_{n-i+1}^{-x_{i}} \operatorname{Sp}_{\left(-x_{n}, \ldots,-x_{1}\right)}(v)=\frac{(-1)^{n} \prod_{j=1}^{n} v_{j}^{-j}}{\operatorname{det}\left(v_{i}^{j}-v_{i}^{-j}\right)_{i, j=1}^{n}}
$$

and use Eq. 24.17 from [17] to give a more explicit expression for the limiting constant

$$
\operatorname{det}\left(v_{i}^{j}-v_{i}^{-j}\right)_{i, j=1}^{n}=(-1)^{n} \prod_{i<j}\left(v_{i}-v_{j}\right) \prod_{1 \leq i \leq j \leq n}\left(1-v_{i} v_{j}\right) \prod_{j=1}^{n} v_{j}^{-n} .
$$

We conclude that

$$
\lim _{x_{i}-x_{i+1} \rightarrow-\infty} \kappa_{C} \prod_{i=1}^{n} v_{n-i+1}^{-x_{i}} \operatorname{Sp}_{\left(-x_{n}, \ldots,-x_{1}\right)}(v)=1 .
$$


In the case $0<v_{n}<\ldots<v_{1}<1$ the process $\left(Z^{\left(v_{n}\right)}(t), \ldots, Z^{\left(v_{1}\right)}(t)\right)$ almost surely has $Z_{i}^{\left(v_{i}\right)} \rightarrow-\infty$ for each $i=1, \ldots, n$ and $Z_{i}^{\left(v_{i}\right)}-Z_{i+1}^{\left(v_{i+1}\right)} \rightarrow-\infty$ for $i=1, \ldots, n-1$. Therefore the above specifies the boundary behaviour of $\kappa_{A} h_{A}$ and $\kappa_{C} h_{C}$.

Suppose that $(h, T)$ either equals $\left(\kappa_{A} h_{A}, T_{A}\right)$ or $\left(\kappa_{C} h_{C}, T_{C}\right)$ and let $Z_{t}^{*}$ denote $\left(Z^{\left(v_{n}\right)}(t), \ldots, Z^{\left(v_{1}\right)}(t)\right)$ killed at the instant it leaves $W^{n}$ or $W_{<0}^{n}$. Then $\left(h\left(Z_{t}^{*}\right)\right)_{t \geq 0}$ is a bounded martingale and converges almost surely and in $L^{\overline{1}}$ to a random variable $\mathcal{Y}$. From the boundary behaviour specified above, $\mathcal{Y}$ equals 1 if $T=\infty$ and equals zero otherwise almost surely. Using this in the $L^{1}$ convergence shows that $h(x)=$ $\lim _{t \rightarrow \infty} E_{x}\left(h\left(Z_{t}^{*}\right)\right)=P_{x}(T=\infty)$.

From this we can prove the second equality in law in Theorem 1.1 for a particular choice of rates. Suppose $0<v_{n}<\ldots<v_{1}<1$ which ensures that all of the following events have strictly positive probabilities, and let $x \in W_{\leq 0}^{n}$. Then

$$
\begin{aligned}
P_{\left(x_{1}, \ldots, x_{n}\right)}\left(\sup _{t \geq 0} Z_{n}^{\dagger}<0\right) & =\frac{P_{\left(x_{1}, \ldots, x_{n}\right)}\left(T_{C}=\infty\right)}{P_{\left(x_{1}, \ldots, x_{n}\right)}\left(T_{A}=\infty\right)} \\
& =\frac{\kappa_{C} h_{C}(x)}{\kappa_{A} h_{A}(x)} \\
& =\prod_{1 \leq i \leq j \leq n}\left(1-v_{i} v_{j}\right) \frac{\operatorname{Sp}_{\left(-x_{n}, \ldots,-x_{1}\right)}(v)}{\mathrm{S}_{x}(v)} .
\end{aligned}
$$

Let $\left(x_{1}, \ldots, x_{n}\right) \rightarrow(-\eta, \ldots,-\eta)$ and shift co-ordinates by $\eta$. Then

$$
P_{0}\left(\sup _{t \geq 0} Z_{n}^{\dagger} \leq \eta\right)=P_{-\eta}\left(\sup _{t \geq 0} Z_{n}^{\dagger} \leq 0\right)=\prod_{1 \leq i \leq j \leq n}\left(1-v_{i} v_{j}\right) \prod_{i=1}^{n} v_{i}^{\eta} \operatorname{Sp}_{\eta^{(n)}}(v)
$$

by using that $S_{\left(x_{1}, \ldots, x_{n}\right)}(v) \rightarrow \prod_{i=1}^{n} v_{i}^{-\eta}$ and the notation $\eta^{(n)}=(\eta, \ldots, \eta)$. We compare this to Corollary 4.2 of [5] which in our notation states that

$$
P(G(1,1) \leq \eta)=\prod_{1 \leq i \leq j \leq n}\left(1-v_{i} v_{j}\right) \prod_{i=1}^{n} v_{i}^{\eta} \operatorname{Sp}_{\eta^{(n)}}(v) .
$$

Equation (2.6) and (2.7) prove the second equality in law in Theorem 1.1 for $0<v_{n}<$ $\ldots<v_{1}<1$. This can be extended to all distinct rates with $v_{i}<1$ for each $i=1, \ldots, n$ by observing that the law of the process $\left(Z_{n}^{\dagger}(t)\right)_{t \geq 0}$ is invariant under permutations of the $v_{i}$. In particular, this holds for $\sup _{t \geq 0} Z_{n}^{\dagger}(t)$ and also holds for $G(1,1)$ from (2.7).

\subsection{Time reversal}

Up to this point we have considered PushASEP with a wall as a continuous-time Markov chain with a unique stationary distribution. We now show that PushASEP with a wall started from $(0, \ldots, 0)$ has an interpretation as semi-discrete last passage percolation times in a environment constructed from $2 n$ Poisson point processes. The stationary distribution can be recovered by taking a limit as $t \rightarrow \infty$. In particular,

$$
\left(Y_{k}(t)\right)_{k=1}^{n}=\left(\sup _{0 \leq t_{0} \leq t_{1} \leq \ldots \leq t_{k}=t} \sum_{i=1}^{k}\left(Z_{i}^{\left(v_{i}\right)}\left(t_{i}\right)-Z_{i}^{\left(v_{i}\right)}\left(t_{i-1}\right)\right)\right)_{k=1}^{n}
$$

where the $Z_{i}^{\left(v_{i}\right)}$ are continuous-time random walks defined above. In terms of the semidiscrete last passage percolation formulation, it is natural to think of $Z_{i}^{\left(v_{i}\right)}$ as a difference of two Poisson point processes, one acting as a reward (at rate $v_{i}$ ) and one as a penalty (at rate $v_{i}^{-1}$ ). 
In the proof of (2.8) we will denote the right hand side of (2.8) by $\left(U_{k}(t)\right)_{k=1}^{n}$. We check that the evolution of this process is PushASEP with a wall. When $n=1, U_{1}(t)=$ $\sup _{0 \leq t_{0} \leq t}\left(Z_{1}^{\left(v_{1}\right)}(t)-Z_{1}^{\left(v_{1}\right)}\left(t_{0}\right)\right)$ and this evolves as PushASEP with a wall with one particle started from zero. For the inductive step we note that adding in the $n$-th particle to $\left(U_{k}(t)\right)_{k=1}^{n}$ does not affect the evolution of the first $(n-1)$ particles. Therefore we only need to consider the $n$-th particle given by

$$
U_{n}(t)=\sup _{0 \leq s \leq t}\left(Z_{n}^{\left(v_{n}\right)}(t)-Z_{n}^{\left(v_{n}\right)}(s)+Y_{n-1}(s)\right)
$$

where $Y_{n-1}$ is the $(n-1)$-th particle in PushASEP with a wall. If $U_{n}>Y_{n-1}$ then the suprema in (2.9) is attained with a choice $s<t$ and $U_{n}$ jumps right or left whenever $Z_{n}^{\left(v_{n}\right)}$ does. If $U_{n}=Y_{n-1}$ then at least one of the (possibly non-unique) maximisers of the supremum in (2.9) involves $s=t$. This means that if $Z_{n}^{\left(v_{n}\right)}$ jumps to the right then $U_{n}$ jumps to the right; if $Y_{n-1}$ jumps to the right then $U_{n}$ jumps to the right (this is the pushing interaction); and if $Z_{n}^{\left(v_{n}\right)}$ jumps to the left then $U_{n}$ is unchanged (this is the blocking interaction). Therefore $U_{n}$ defined by (2.9) follows the dynamics of the $n$-th particle in PushASEP with a wall started from the origin. Therefore (2.8) follows inductively.

Equation 2.8 has a similar form to Proposition 2.1 and this along with time reversal establishes the following connection, see $[9,16]$ for a similar argument in a Brownian context.

Proposition 2.4. Let $Y_{n}^{*}$ be distributed as the top particle in PushASEP with a wall in its invariant measure and $Z_{n}^{\dagger}$ be the top particle in the ordered random walk with $Q$-matrix given by (2.3) and started from the origin. Then

$$
Y_{n}^{*} \stackrel{d}{=} \sup _{t \geq 0} Z_{n}^{\dagger}(t)
$$

Proof. For any fixed $t$, we let $t-u_{i}=t_{k-i}$ and use time reversal of continuous-time random walks $\left(Z_{i}^{\left(v_{i}\right)}(t)-Z_{i}^{\left(v_{i}\right)}(t-s)\right)_{s \geq 0} \stackrel{d}{=}\left(Z_{n-i+1}^{\left(v_{i}\right)}(s)\right)_{s \geq 0}$ to establish that

$$
\begin{aligned}
\left(Y_{k}(t)\right)_{k=1}^{n} & =\left(\sup _{0 \leq t_{0} \leq \ldots \leq t_{k}=t} \sum_{i=1}^{k}\left(Z_{i}^{\left(v_{i}\right)}\left(t_{i}\right)-Z_{i}^{\left(v_{i}\right)}\left(t_{i-1}\right)\right)\right)_{k=1}^{n} \\
& =\left(\sup _{0=u_{0} \leq \ldots \leq u_{k} \leq t} \sum_{i=1}^{k}\left(Z_{i}^{\left(v_{i}\right)}\left(t-u_{k-i}\right)-Z_{i}^{\left(v_{i}\right)}\left(t-u_{k-i+1}\right)\right)\right)_{k=1}^{n} \\
& \stackrel{d}{=}\left(\sup _{0=u_{0} \leq \ldots \leq u_{k} \leq t} \sum_{i=1}^{k}\left(Z_{n-i+1}^{\left(v_{i}\right)}\left(u_{k-i+1}\right)-Z_{n-i+1}^{\left(v_{i}\right)}\left(u_{k-i}\right)\right)\right)_{k=1}^{n}
\end{aligned}
$$

The final step uses that the summands are independent and are increments of the forwards and backwards in time processes in the time reversal above which are equal as processes. The equality in law of the largest co-ordinates, relabelling the sum from $i$ to $n-i+1$ and comparing with Proposition 2.1 part (ii) shows that $Y_{n}(t) \stackrel{d}{=} \sup _{0 \leq s \leq t} Z_{n}^{\dagger}(s)$. In particular, letting $t \rightarrow \infty$ completes the proof.

Lemma 2.5. The distribution of $\left(Y_{1}^{*}, \ldots, Y_{n}^{*}\right)$ is continuous in $\left(v_{1}, \ldots, v_{n}\right)$ on the set $(0,1)^{n}$.

Proof. We will use the representation for $\left(Y_{1}^{*}, \ldots, Y_{n}^{*}\right)$ obtained by relabelling the sum $i$ to $k-i+1$ in (2.10) and letting $t \rightarrow \infty$,

$$
\left(Y_{k}^{*}\right)_{k=1}^{n} \stackrel{d}{=}\left(\sup _{0=t_{0} \leq \ldots \leq t_{k}<\infty} \sum_{i=1}^{k}\left(Z_{n-k+i}^{\left(v_{k-i+1}\right)}\left(t_{k-i+1}\right)-Z_{n-k+i}^{\left(v_{k-i+1}\right)}\left(t_{k-i}\right)\right)\right)_{k=1}^{n} .
$$


We fix $\epsilon>0$ and construct realisations of $Z_{i}^{\left(v_{n-i+1}\right)}$ for all $\epsilon<v_{i}<1-\epsilon$ on the same probability space. To achieve this we define $2 n$ independent marked Poisson point process $R_{1}, \ldots, R_{n}$ and $L_{1}, \ldots, L_{n}$ on $\mathbb{R}_{\geq 0} \times[0,1]$ which will dictate the rightwards and leftward jumps respectively of $Z_{i}^{\left(v_{n-i+1}\right)}$. For each $i=1, \ldots, n$, the marked Poisson point process $R_{i}$ and $L_{i}$ consist of points $\left(t_{k}, w_{k}\right)_{k \geq 1}$ and $\left(\bar{t}_{k}, \bar{w}_{k}\right)_{k \geq 1}$ where $\left(t_{k}\right)_{k \geq 1}$ and $\left(\bar{t}_{k}\right)_{k \geq 1}$ are the points of a Poisson point process of rate 1 and $1 / \epsilon$ respectively on $\mathbb{R}_{\geq 0}$. The $w_{i}$ and $\bar{w}_{i}$ are uniform random variables on the interval $[0,1]$ which are independent of each other and $\left(t_{i}\right)_{i \geq 1},\left(\bar{t}_{i}\right)_{i \geq 1}$.

We define $R_{i}^{(v)}$ to be the subset of $\left(t_{i}, w_{i}\right)_{i \geq 1}$ with $w_{i}>1-v$ and $L_{i}^{(1 / v)}$ to be the subset of $\left(\bar{t}_{i}, \bar{w}_{i}\right)_{i \geq 1}$ with $\bar{w}_{i}>1-\epsilon / v$. The projection onto the first co-ordinate of $R_{i}^{\left(v_{i}\right)}$ and $L_{i}^{\left(v_{i}\right)}$ give independent Poisson point process of rate $v_{i}$ and $1 / v_{i}$ respectively which define coupled realisations of $Z_{i}^{\left(v_{n-i+1}\right)}$ for any choice of $\epsilon<v<1-\epsilon$.

Almost surely, the suprema on the right hand side of Proposition 2.8 part (ii) all stablise (after some random time uniform over $\left(v_{1}, \ldots, v_{n}\right) \in(\epsilon, 1-\epsilon)^{n}$ ). For any realisation of the marked Poisson point processes, the right hand side of Proposition 2.8 part (ii) is continuous in $\left(v_{1}, \ldots, v_{n}\right)$ except at $\left(1-w_{i}\right)_{i \geq 1}$ and $\left(\epsilon /\left(1-w_{i}\right)\right)_{i \geq 1}$. Therefore the distribution of the right hand side of part (ii) of Proposition 2.8 is continuous in $\left(v_{1}, \ldots, v_{n}\right)$ on the set $(\epsilon, 1-\epsilon)^{n}$, and hence so is the distribution of $\left(Y_{1}^{*}, \ldots, Y_{n}^{*}\right)$. As $\epsilon$ is arbitrary this completes the proof.

Proof of Theorem 1.1. Proposition 2.4 is the first equality in law. At the end of Section 2.1 we proved the second equality for distinct $0<v_{1}, \ldots, v_{n}<1$. Lemma 2.5 allows us to remove the constraint that the $v_{i}$ are distinct.

\section{Push ASEP with a wall}

\subsection{Transition probabilities}

We give a more explicit definition of Push-ASEP with a wall at the origin as a continuous-time Markov chain $(Y(t))_{t \geq 0}=\left(Y_{1}(t), \ldots, Y_{n}(t)\right)_{t \geq 0}$ taking values in $W_{>0}^{n}=$ $\left\{\left(y_{1}, \ldots, y_{n}\right): y_{i} \in \mathbb{Z}\right.$ and $\left.0 \leq y_{1} \leq y_{2} \ldots \leq y_{n}\right\}$. We use $e_{i}$ to denote the vector taking value 1 in position $i$ and zero otherwise. The transition rates of $Y$ are defined for $y, y+e_{i}+\ldots+e_{j} \in W_{\geq 0}^{n}$ and $i \leq j$ by

$$
q\left(y, y+e_{i}+e_{i+1}+\ldots+e_{j}\right)=v_{i} 1_{\left\{y_{i}=y_{i+1}=\ldots=y_{j}<y_{j+1}\right\}}
$$

with the notation $y_{n+1}=\infty$ and for $y \in W_{\geq 0}^{n}$ by

$$
q\left(y, y-e_{i}\right)=v_{i}^{-1} 1_{\left\{y-e_{i} \in W_{\geq 0}^{n}\right\}} .
$$

All other transition rates equal zero. We note that in [8] the particles were strictly ordered, whereas it is convenient for us to consider a weakly ordered system; these systems can be related by a co-ordinate change $x_{j} \rightarrow x_{j}+j-1$.

To describe the transition probabilities we first introduce the operators acting on functions $f: \mathbb{Z} \rightarrow \mathbb{R}$ with $v>0$,

$$
D^{(v)} f(u)=f(u)-v f(u-1), \quad J^{(v)} f(u)=\sum_{j=u}^{\infty} v^{u-j} f(j),
$$

where we will always apply $J^{(v)}$ to functions with superexponential decay at infinity. We use $D^{\left(v_{1}, \ldots, v_{n}\right)}=D^{\left(v_{1}\right)} \ldots D^{\left(v_{n}\right)}$ and $J^{\left(v_{1}, \ldots, v_{n}\right)}=J^{\left(v_{1}\right)} \ldots J^{\left(v_{n}\right)}$ as notation for concatenated operators and $D_{u}^{(v)}, J_{u}^{(v)}$ to specify a variable $u$ on which the operators act. 
We recall Siegmund duality for birth-death processes, see for example [11, 13]. Let $\left(X_{t}\right)_{t \geq 0}$ denote a birth-death process on the state space $\mathbb{Z}_{\geq 0}$ with transition rates:

$$
i \rightarrow i+1 \text { rate } \lambda_{i} \text { for } i \geq 0, \quad i \rightarrow i-1 \text { rate } \mu_{i} \text { for } i \geq 1 .
$$

Let $\left(X_{t}^{*}\right)_{t \geq 0}$ denote a birth-death process on the state space $\mathbb{Z}_{\geq-1}$ with transition rates

$$
i \rightarrow i+1 \text { rate } \mu_{i+1} \text { for } i \geq 0, \quad i \rightarrow i-1 \text { rate } \lambda_{i} \text { for } i \geq 0 .
$$

The process $X$ has a reflecting boundary at zero while $X^{*}$ is absorbed at -1 . Under suitable conditions on the rates, see [13], which hold in the case of interest to us: $\lambda_{i}=v_{1}$ for $i \geq 0$ and $\mu_{i}=v_{1}^{-1}$ for $i \geq 1$, Siegmund duality states that

$$
P_{x}\left(X_{t} \leq y\right)=P_{y}\left(X_{t}^{*} \geq x\right) \quad \text { for all } t \geq 0, \quad x, y \in \mathbb{Z}_{\geq 0}
$$

We can find the transition probabilities for $X^{*}$ by solving the Kolmogorov forward equation. We define for any $t \geq 0$ and $x, y \in \mathbb{Z}$,

$$
\psi_{t}(x, y)=\frac{1}{2 \pi i} \oint_{\Gamma_{0}} \frac{d z}{z}\left(z^{y-x}-z^{x+y+2}\right) e^{t(z+1 / z)}
$$

where $\Gamma_{0}$ denotes the unit circle oriented anticlockwise. The transition probabilities of $X^{*}$ are given for $x \in \mathbb{Z}_{\geq 0}$ and $t \geq 0$ by $P_{x}\left(X_{t}^{*}=y\right)=v_{1}^{y-x} e^{-t\left(v_{1}+1 / v_{1}\right)} \psi_{t}(x, y)$ for $y \in \mathbb{Z}_{\geq 0}$ and $P_{x}\left(X_{t}^{*}=-1\right)=1-\sum_{y \geq 0} v_{1}^{y-x} e^{-t\left(v_{1}+1 / v_{1}\right)} \psi_{t}(x, y)$.

By using Siegmund duality, the transition probabilities of PushASEP with a wall with a single particle (which is an M/M/1 queue) are given by

$$
v_{1}^{y-x} e^{-t\left(v_{1}+1 / v_{1}\right)} D_{y}^{\left(1 / v_{1}\right)} J_{x}^{\left(v_{1}\right)} \psi_{t}(x, y) \quad \text { for all } t \geq 0, \quad x, y \in \mathbb{Z}_{\geq 0}
$$

The purpose of the above is that this now provides a form which is convenient to generalise to $n$ particles. Define for all $t \geq 0$ and $x, y \in \mathbb{Z}^{n}$,

$$
r_{t}(x, y)=\prod_{k=1}^{n} v_{k}^{y_{k}-x_{k}} e^{-t\left(v_{k}+1 / v_{k}\right)} \operatorname{det}\left(F_{i j}\left(t ; x_{i}+i-1, y_{j}+j-1\right)\right)_{i, j=1}^{n}
$$

with

$$
F_{i j}\left(t ; x_{i}+i-1, y_{j}+j-1\right)=D_{y_{j}}^{\left(1 / v_{1} \ldots 1 / v_{j}\right)} J_{x_{i}}^{\left(v_{1} \ldots v_{i}\right)} \psi_{t}\left(x_{i}+i-1, y_{j}+j-1\right) .
$$

Proposition 3.1. The transition probabilities of $\left(Y_{1}(t), \ldots, Y_{n}(t)\right)_{t \geq 0}$ are given by $r_{t}(x, y)$ for $x, y \in W_{\geq 0}^{n}$.

The transition probabilities for PushASEP in the absence of a wall were found in [8] and related examples have been found in [30, 32]. Our proof follows the ideas in [8].

Proof. Observe that for all $u, w \in \mathbb{Z}$,

$$
\frac{d \psi}{d t}=\psi_{t}(u, w+1)+\psi_{t}(u, w-1)
$$

and therefore for all $x, y \in W^{n}$,

$$
\frac{d r}{d t}=\sum_{k=1}^{n} v_{k}^{-1} r_{t}\left(x, y+e_{k}\right)+v_{k} r_{t}\left(x, y-e_{k}\right)-\left(v_{k}+v_{k}^{-1}\right) r_{t}(x, y) .
$$

We note that $y \pm e_{k}$ may be outside of the set $W_{\geq 0}^{n}$ but that $r$ has been defined for all $x, y \in \mathbb{Z}^{n}$. The proof will involve showing that the terms involving $y \pm e_{k} \notin W_{\geq 0}^{n}$ in (3.3) 
can be replaced, using identities for $r$, by terms corresponding to the desired pushing and blocking interactions.

An important role is played by the identity, that if $y_{j}=y_{j+1}$ then

$$
v_{j}^{-1} r_{t}\left(x, y+e_{j}\right)=v_{j+1}^{-1} r_{t}(x, y) .
$$

This can be proved by showing that the difference of the two sides is equal to

$$
\prod_{k=1}^{n} v_{k}^{y_{k}-x_{k}} e^{-t\left(v_{k}+1 / v_{k}\right)} \operatorname{det}\left(A_{i j}\right)_{i, j=1}^{n}
$$

where the relevant columns of $A$ are the $k$-th and $(k+1)$-th which have entries for each $i=1, \ldots, n$ given by

$$
A_{i k}=D_{y_{k+1}}^{\left(1 / v_{k+1}\right)} F_{i k}\left(x_{i}+i-1, y_{k+1}+k\right), \quad A_{i k+1}=F_{i k+1}\left(x_{i}+i-1, y_{k+1}+k\right) .
$$

These two columns are equal which proves (3.4).

We first consider the terms in (3.3) with $y-e_{k} \notin W_{\geq 0}^{n}$ and $y_{k}>0$ which corresponds to right jumps with a pushing interaction. Denote by $m(k)$ the minimal index such that $y_{m(k)}=y_{m(k+1)}=\ldots=y_{k}$. Then by iteratively applying the identity (3.4) we obtain

$$
v_{k} r_{t}\left(x, y-e_{k}\right)=v_{k-1} r_{t}\left(x, y-e_{k-1}-e_{k}\right)=\ldots=v_{m(k)} r_{t}\left(x, y-e_{m(k)}-\ldots-e_{k-1}-e_{k}\right) .
$$

This shows that,

$$
\begin{aligned}
& \sum_{k=1}^{n} v_{k} r_{t}\left(x, y-e_{k}\right) 1_{\left\{y_{m(k-1)}<y_{m(k)}=\ldots=y_{k}\right\}}-v_{k} r_{t}(x, y) \\
& \quad=\sum_{k=1}^{n} v_{m(k)} r_{t}\left(x, y-e_{m(k)}-\ldots-e_{k-1}-e_{k}\right) 1_{\left\{y_{m(k-1)}<y_{m(k)}=\ldots=y_{k}\right\}}-v_{k} r_{t}(x, y)
\end{aligned}
$$

where $y_{0}:=0$. We note that $y-e_{m(k)}-\ldots-e_{k-1}-e_{k} \in W_{\geq 0}^{n}$ whenever $y_{m(k-1)}<y_{m(k)}=$ $\ldots=y_{k}$ holds.

We next consider the terms in (3.3) with $y+e_{k} \notin W_{\geq 0}^{n}$ which will correspond to blocking interactions. This means that $y_{k}=y_{k+1}$ and using (3.4) shows that

$$
\sum_{k=1}^{n-1} v_{k}^{-1} 1_{\left\{y_{k}=y_{k+1}\right\}} r_{t}\left(x, y+e_{k}\right)=\sum_{k=2}^{n} v_{k}^{-1} 1_{\left\{y_{k-1}=y_{k}\right\}} r_{t}(x, y) .
$$

Therefore

$$
\begin{array}{r}
\sum_{k=1}^{n} v_{k}^{-1} r_{t}\left(x, y+e_{k}\right)-v_{k}^{-1} r_{t}(x, y)=-v_{1}^{-1} r_{t}(x, y)-\sum_{k=2}^{n} v_{k}^{-1}\left(1-1_{\left\{y_{k-1}=y_{k}\right\}}\right) r_{t}(x, y) \\
+v_{n}^{-1} r_{t}\left(x, y+e_{n}\right)+\sum_{k=1}^{n-1} v_{k}^{-1}\left(1-1_{\left\{y_{k}=y_{k+1}\right\}}\right) r_{t}\left(x, y+e_{k}\right) .
\end{array}
$$

We note that $y+e_{k} \in W_{\geq 0}^{n}$ whenever $\left(1-1_{\left\{y_{k}=y_{k+1}\right\}}\right) \neq 0$.

The final terms we need to consider in (3.3) are those with $y-e_{k} \notin W_{\geq 0}^{n}$ and $y_{k}=0$ which correspond to left jumps which are suppressed by the wall. If $y_{1}=\ldots=y_{k}=0$ for some $k>1$, then

$$
r_{t}\left(x, y-e_{k}\right)=v_{k}^{-1} \prod_{j=1}^{n} v_{j}^{y_{j}-x_{j}} e^{-t\left(v_{j}+1 / v_{j}\right)} \operatorname{det}\left(B_{i j}\right)_{i, j=1}^{n}
$$


for a matrix $B$ where the relevant entries of $B$ are the columns indexed by $1, \ldots, k$. The first column has entries $B_{i 1}=J_{x_{i}}^{\left(v_{1} \ldots v_{i}\right)}\left(\psi\left(x_{i}-i+1,0\right)-v_{1}^{-1} \psi\left(x_{i}-i+1,-1\right)\right)$ which simplifies to $B_{i 1}=J_{x_{i}}^{\left(v_{1} \ldots v_{i}\right)} \psi\left(x_{i}-i+1,0\right)$ by the fact that $\psi(\cdot,-1)=0$. The columns indexed by $j=2, \ldots, k-1$ can be simplified to $B_{i j}=J_{x_{i}}^{\left(v_{1} \ldots v_{i}\right)} \psi\left(x_{i}-i+1, j-1\right)$ by using $\psi(\cdot,-1)=0$ and column operations. Using this argument for the $k$-th column and that we consider the vector $y-e_{k}$, we observe that the $k$-th column is a linear combination of columns $1, \ldots, k-1$ and hence $r_{t}\left(x, y-e_{k}\right)=0$ if $y_{k}=0$ for any $k>1$.

The remaining case is when $0=y_{1}<y_{2}$ and we show that

$$
v_{1} r_{t}\left(x, y-e_{1}\right)-v_{1}^{-1} r_{t}(x, y)=0 .
$$

This follows from multilinearity of the determinants involved in the definition of $r$ and using $\psi(\cdot,-1)=0$,

$$
D^{\left(1 / v_{1}\right)} \psi\left(x_{i},-1\right)-v_{1}^{-1} D^{\left(1 / v_{1}\right)} \psi_{t}\left(x_{i}, 0\right)=-v_{1}^{-1} \psi\left(x_{i},-2\right)-v_{1}^{-1} \psi_{t}\left(x_{i}, 0\right)=0 .
$$

Therefore

$$
\sum_{k=1}^{n} v_{k} r_{t}\left(x, y-e_{k}\right) 1_{\left\{0=y_{1}=\ldots=y_{k}\right\}}=v_{1}^{-1} 1_{\left\{y_{1}=0\right\}} r_{t}(x, y) .
$$

We combine (3.3), (3.5), (3.6) and (3.7) to obtain that

$$
\begin{aligned}
\frac{d r}{d t} & =\sum_{k=1}^{n} v_{m(k)} r_{t}\left(x, y-e_{m(k)}-\ldots-e_{k-1}-e_{k}\right) 1_{\left\{y_{m(k-1)}<y_{m(k)}=\ldots=y_{k}\right\}}-\sum_{k=1}^{n} v_{k} r_{t}(x, y) \\
& -v_{1}^{-1}\left(1-1_{\left\{y_{1}=0\right\}}\right) r_{t}(x, y)-\sum_{k=2}^{n} v_{k}^{-1}\left(1-1_{\left\{y_{k-1}=y_{k}\right\}}\right) r_{t}(x, y) \\
& +v_{n}^{-1} r_{t}\left(x, y+e_{n}\right)+\sum_{k=1}^{n-1} v_{k}^{-1}\left(1-1_{\left\{y_{k}=y_{k+1}\right\}}\right) r_{t}\left(x, y+e_{k}\right)
\end{aligned}
$$

We now consider the initial condition.

$$
r_{0}(x, y)=\prod_{k=1}^{n} v_{k}^{y_{k}-x_{k}} \operatorname{det}\left(F_{i j}\left(0 ; x_{i}+i-1, y_{j}+j-1\right)\right)_{i, j=1}^{n}
$$

where

$$
F_{i j}\left(0 ; x_{i}+i-1, y_{j}+j-1\right)=D_{y_{j}}^{\left(1 / v_{1} \ldots 1 / v_{j}\right)} J_{x_{i}}^{\left(v_{1} \ldots v_{i}\right)} \psi_{0}\left(x_{i}+i-1, y_{j}+j-1\right)
$$

and $\psi_{0}(u, w)=1_{\{w-u=0\}}$ for $u, w \geq 0$ depends only on the difference $w-u$ and we will view this as a function of $w-u$. For any function $f: \mathbb{Z} \rightarrow \mathbb{R}$ and $u, w \in \mathbb{Z}, r>0$

$$
D_{w}^{(1 / r)} J_{u}^{(r)} f(w-u)=D_{w}^{(1 / r)}\left(\sum_{k=u}^{\infty} r^{u-k} f(w-k)\right)=f(w-u) .
$$

Therefore the top-left entry in the matrix defining $r_{0}$ equals $1_{\left\{y_{1}=x_{1}\right\}}$. Suppose $y_{1}>x_{1}$ and observe that if a function $g$ has $g(u)=0$ for $u>0$, then for any $j=1, \ldots, n$ we have $D_{u}^{\left(1 / v_{2} \ldots 1 / v_{j}\right)} g(u+j-1)=0$ for $u>0$. This shows that when $y_{1}>x_{1}$ the top row of the matrix defining $r_{0}$ equals zero. In a similar manner, when $y_{1}<x_{1}$ the first column in the matrix defining $r_{0}$ is zero. Therefore

$$
r_{0}(x, y)=1_{\left\{x_{1}=y_{1}\right\}} \prod_{k=2}^{n} v_{k}^{y_{k}-x_{k}} \operatorname{det}\left(F_{i j}\left(0 ; x_{i}+i-1, y_{j}+j-1\right)\right)_{i, j=2}^{n}
$$


and using (3.12) the entries of the matrix in (3.13) have the same form as the entries of the matrix in (3.11) but with $n-1$ particles. Continuing inductively,

$$
r_{0}(x, y)=\prod_{k=1}^{n} 1_{\left\{x_{k}=y_{k}\right\}} .
$$

Therefore $r_{t}(x, y)$ satisfies the Kolmogorov forward equations (3.8) and (3.14) corresponding to the process $(Y(t))_{t \geq 0}$. These equations have a unique solution given by the transition probabilities of $(Y(t))_{t \geq 0}$ because the process does not explode.

Lemma 3.2. Let $\left(f_{i}\right)_{i=1}^{n}$ and $\left(g_{j}\right)_{j=1}^{n}$ be functions $\mathbb{Z}_{\geq-1} \rightarrow \mathbb{R}$ such that $g_{j}$ decays superexponentially while $f_{i}$ grows at most exponentially at infinity.

(i) Suppose further that $f_{i}(-1)=0$ for each $i=1, \ldots, n$,

$$
\begin{aligned}
\sum_{x \in W_{\geq 0}^{n}} \operatorname{det}\left(D^{\left(1 / v_{1} \ldots 1 / v_{j}\right)} f_{i}\left(x_{j}+j-1\right)\right)_{i, j=1}^{n} \operatorname{det}\left(J^{\left(v_{1} \ldots v_{i}\right)} g_{j}\left(x_{i}+i-1\right)\right)_{i, j=1}^{n} & \\
& =\operatorname{det}\left(\sum_{u \geq 0} f_{i}(u) g_{j}(u)\right)_{i, j=1}^{n} .
\end{aligned}
$$

(ii) With no extra conditions and the notation $D^{\emptyset}=I d$,

$$
\begin{aligned}
\sum_{x \in W_{\geq 0}^{n}} \operatorname{det}\left(D^{\left(1 / v_{2} \ldots 1 / v_{j}\right)} f_{i}\left(x_{j}+j-1\right)\right)_{i, j=1}^{n} \operatorname{det}\left(J^{\left(v_{2} \ldots v_{i}\right)} g_{j}\left(x_{i}+i-1\right)\right)_{i, j=1}^{n} & \\
& =\operatorname{det}\left(\sum_{u \geq 0} f_{i}(u) g_{j}(u)\right)_{i, j=1}^{n} .
\end{aligned}
$$

Proof. The proof is similar to Lemma 2 in [16] and so we give a description of the proof and refer to [16] which carries out some of the steps more explicitly. We prove (i) first and (ii) is almost identical.

We first observe that

$$
\sum_{u=a}^{b}\left(D^{(1 / v)} f\right)(u+1)\left(J^{(v)} g\right)(u+1)=\sum_{u=a}^{b} f(u) g(u)+f(b+1)\left(J^{(v)} g\right)(b+1)-f(a)\left(J^{(v)} g\right)(a) .
$$

We apply (3.15) repeatedly to show that

$$
\begin{aligned}
\sum_{x \in W_{\geq 0}^{n}} \operatorname{det}\left(D^{\left(1 / v_{1} \ldots 1 / v_{j}\right)} f_{i}\left(x_{j}+j-1\right)\right)_{i, j=1}^{n} \operatorname{det}\left(J^{\left(v_{1} \ldots v_{i}\right)} g_{j}\left(x_{i}+i-1\right)\right)_{i, j=1}^{n} \\
=\sum_{x \in W_{\geq 0}^{n}} \operatorname{det}\left(f_{i}\left(x_{j}-1\right)\right)_{i, j=1}^{n} \operatorname{det}\left(g_{j}\left(x_{i}-1\right)\right)_{i, j=1}^{n} .
\end{aligned}
$$

The general procedure is to use a Laplace expansion of the determinants on the left hand side, apply (3.15) with a particular choice of variable and parameter, and then reconstruct the result as a sum of three determinants. A key property is that all of the boundary terms in (3.15) will end up contributing zero.

The first application of this procedure is with the parameter $v_{n}$, variable $x_{n}$ and summing $x_{n}$ from $x_{n-1}$ to infinity. This shows that the left hand side of (3.16) equals a sum of three terms which all take the form:

$$
\sum_{\Sigma} \operatorname{det}\left(A_{i j}\right)_{i, j=1}^{n} \operatorname{det}\left(B_{i j}\right)_{i, j=1}^{n} .
$$


In the first term, $\Sigma=\left\{\left(x_{1}, \ldots, x_{n}\right) \in W_{\geq 0}^{n}\right\}$. The $A_{i j}$ are given by the entries of the first matrix on the left hand side of (3.16) except with the application of $D^{\left(1 / v_{n}\right)}$ in the $n$-th column removed and the argument $x_{n}+n-1$ replaced by $x_{n}+n-2$. The $B_{i j}$ are given by the entries of the second matrix on the left hand side of (3.16) except with the application of $J^{\left(1 / v_{n}\right)}$ in the $n$-th row removed and the argument $x_{n}+n-1$ replaced by $x_{n}+n-2$. There are two boundary terms which have $\Sigma=\left\{\left(x_{1}, \ldots, x_{n-1}\right) \in W_{n-1}^{+}\right\}$and are evaluated at $x_{n}=x_{n-1}$ and $x_{n}=\infty$. These terms are both zero: when evaluated at $x_{n}=x_{n-1}$ two columns in $A_{i j}$ are equal, and the boundary term at infinity vanishes due to the growth and decay conditions imposed on $f$ and $g$.

We continue this process of using (3.15) with the following orders of parameters and variables: $\left(x_{n}, v_{n}\right),\left(x_{n-1}, v_{n-1}\right), \ldots,\left(x_{1}, v_{1}\right),\left(x_{n-1}, v_{n}\right),\left(x_{n-2}, v_{n-1}\right), \ldots,\left(x_{2}, v_{1}\right),\left(x_{n}, v_{1}\right)$. For each $j=2, \ldots, n-1$ the sum in (3.15) when applied to the $x_{j}$ variable is from $x_{j-1}$ to $x_{j+1}$ and all boundary terms are zero. In the generic case, the boundary term corresponding to the upper limit of summation is evaluated at $x_{j}=x_{j+1}+1$ and is zero because two rows in the determinant of $B_{i j}$ are equal. When (3.15) is applied to the $x_{1}$ variable the sum is from 0 to $x_{2}$ and the boundary term at zero vanishes by the condition that $f_{i}(-1)=0$ for each $i=1, \ldots, n$.

This proves (3.16) and we apply the Cauchy-Binet (or Andréief) identity to the right hand side of (3.16) to complete the proof of part (i).

Part (ii) is identical except that we do not apply (3.15) to the $x_{1}$ variable. Thus the condition $f_{i}(-1)=0$ for each $i=1, \ldots, n$ can be omitted.

\subsection{Invariant measure}

Proposition 3.3. Let $\left(Y_{1}^{*}, \ldots, Y_{n}^{*}\right)$ be distributed according to the invariant measure of PUShASEP with a wall and suppose that the rates $0<v_{1}, \ldots, v_{n}<1$ are distinct. Then the probability mass function of $\left(Y_{1}^{*}, \ldots, Y_{n}^{*}\right)$ is given by

$$
\pi\left(x_{1}, \ldots, x_{n}\right)=c_{n} \prod_{k=1}^{n} v_{k}^{x_{k}} \operatorname{det}\left(D^{\left(1 / v_{1} \ldots 1 / v_{j}\right)} \phi_{i}\left(x_{j}+j-1\right)\right)_{i, j=1}^{n}
$$

where $\phi_{i}(x)=v_{i}^{-(x+1)}-v_{i}^{x+1}$ and $c_{n}=\prod_{1 \leq i<j \leq n} \frac{1}{\left(v_{i}-v_{j}\right)} \prod_{j=1}^{n} v_{j}^{n}$.

We note that the Markov chain is irreducible, does not explode and the invariant measure is unique when normalised.

Proof. We use Lemma 3.2, noting that $\phi_{i}(-1)=0$ for each $i$ and that the conditions at infinity are satisfied, to find

$$
\sum_{x \in W_{\geq 0}^{n}} \pi(x) r_{t}(x, y)=c_{n} \prod_{k=1}^{n} v_{k}^{y_{k}} e^{-t\left(v_{k}+1 / v_{k}\right)} \operatorname{det}\left(D_{y_{j}}^{\left(1 / v_{1} \ldots 1 / v_{j}\right)} \sum_{u \geq 0} \phi_{i}(u) \psi_{t}\left(u, y_{j}+j-1\right)\right)_{i, j=1}^{n} .
$$

We recall that $\psi$ is related to the transition probabilities of a process $\left(X_{t}^{*}\right)_{t \geq 0}$ defined through two independent Poisson point processes $N_{t}^{(1)}$ and $N_{t}^{(2)}$ of rate 1 as $X_{t}^{*}=$ $N_{t}^{(1)}-N_{t}^{(2)}$ for all $0 \leq t \leq \tau_{-1}$ and $X_{t}^{*}=-1$ for all $t>\tau_{-1}$, where $\tau_{-1}=\inf \{t \geq 0$ : $\left.N_{t}^{(2)}=N_{t}^{(1)}+1\right\}$. The transition probabilities of $X^{*}$ are given for $\xi \geq 0$ by $P_{\xi}\left(X_{t}^{*}=\right.$ $\eta)=e^{-2 t} \psi_{t}(\xi, \eta)$ for $\eta \geq 0$ and $P_{\xi}\left(X_{t}^{*}=-1\right)=1-\sum_{\eta \geq 0} e^{-2 t} \psi_{t}(\xi, \eta)$. On the other hand, $\left(v^{-\left(X_{t}^{*}+1\right)}-v^{X_{t}^{*}+1}\right) e^{-(v+1 / v) t+2 t}$ is a martingale for $X^{*}$ for any $v>0$. In particular,

$$
\sum_{u \geq 0} \psi_{t}\left(y_{j}+j-1, u\right) \phi_{i}(u) e^{-t\left(v_{i}+1 / v_{i}\right)}=\phi_{i}\left(y_{j}+j-1\right) .
$$


Using this and the fact that $\psi$ is symmetric in the right hand side of the first displayed equation in this proof shows that

$$
\sum_{x \in W_{\geq 0}^{n}} \pi(x) r_{t}(x, y)=c_{n} \prod_{k=1}^{n} v_{k}^{y_{k}} \operatorname{det}\left(D^{\left(1 / v_{1} \ldots 1 / v_{j}\right)} \phi_{i}\left(y_{j}+j-1\right)\right)_{i, j=1}^{n}=\pi(y) .
$$

We defer the proof that $\pi$ is positive and the identification of the normalisation constant. These two properties will follow by identifying $\pi$ as the probability mass function for a vector of last passage percolations times in the proof of Theorem 1.2.

\section{Point-to-line last passage percolation}

Point-to-line last passage percolation can be interpreted as an interacting particle system, where at each time step a new particle is added at the origin and particles interact by pushing particles to the right of them. We define a discrete-time Markov chain denoted $\left(\mathbf{G}^{p l}(k)\right)_{1 \leq k \leq n}$ where $\mathbf{G}^{p l}(k)=\left(G_{1}^{p l}(k), \ldots, G_{k}^{p l}(k)\right)$. The particles are updated between time $k-1$ and time $k$ by sequentially defining $G_{1}^{p l}(k), \ldots, G_{n}^{p l}(k)$ starting with $G_{1}^{p l}(k)=g_{n-k+1, k}$ and then applying the update rule

$$
G_{j}^{p l}(k)=\max \left(G_{j}^{p l}(k-1), G_{j-1}^{p l}(k)\right)+g_{n-k+1, k-j+1}, \quad \text { for } 2 \leq j \leq k
$$

where $\left(g_{j k}\right)_{j, k \geq 1, j+k \leq n+1}$ are an independent collection of geometrically distributed random variables with parameters $1-v_{j} v_{n-k+1}$ and $0<v_{j}<1$ for each $j=1, \ldots, n$. The geometric random variables are defined as $P\left(g_{j k}=u\right)=\left(1-v_{j} v_{n-k+1}\right)\left(v_{j} v_{n-k+1}\right)^{u}$ for all $u \geq 0$.

The initial state is $G_{1}^{p l}=g_{n 1}$. The connection to point-to-line last passage percolation is that the largest particle at time $n$ has the representation

$$
G_{n}^{p l}(n)=\max _{\pi \in \Pi_{n}} \sum_{(i, j) \in \pi} g_{i j}
$$

where $\Pi_{n}^{\text {flat }}$ is the set of directed up-right paths nearest neighbour paths from $(1,1)$ to the line $\{(i, j): i+j=n+1\}$. Moreover, $\mathbf{G}^{p l}(n)$ is the vector on the right hand side of Theorem 1.2. The advantage of this interpretation is that the transition probabilities of $\left(\mathbf{G}^{p l}(k)\right)_{1 \leq k \leq n}$ have a determinantal form and this can be used to find the probability mass function of $\mathbf{G}^{p l}(n)$ as a determinant.

In the context of point-to-point last passage percolation the transition kernel of a Markov chain analogous to the above is given in Theorem 1 of [14]. This can be used to describe the update rule of $\mathbf{G}^{p l}(n-1)$ to $\mathbf{G}^{p l}(n)$ from time $n-1$ to time $n$ by viewing $\mathbf{G}^{p l}(n-1)$ as being extended to an $n$-dimensional vector with zero as the leftmost position. We first define: for functions $f: \mathbb{Z} \rightarrow \mathbb{R}$ with $f(u)=0$ for all $u<0$,

$$
D^{(v)} f(u)=f(u)-v f(u-1), \quad I^{(v)} f(u)=\sum_{j=0}^{u} v^{u-j} f(j) \text { for } u \geq 0
$$

and $I^{(v)} f(u)=0$ for $u<0$. Suppose $p^{-1}=\left(1 / p_{1}, \ldots, 1 / p_{n}\right)$ and for a function $g: \mathbb{Z} \rightarrow \mathbb{R}$ with $g(u)=0$ for $u<0$ define

$$
g_{p^{-1}}^{(i j)}(u)= \begin{cases}D^{\left(1 / p_{i+1} \ldots 1 / p_{j}\right)} g(u) & \text { for } j>i \\ I^{\left(1 / p_{j+1} \ldots 1 / p_{i}\right)} g(u) & \text { for } j<i \\ g(u) & \text { for } j=i .\end{cases}
$$


Lemma 4.1 (Dieker, Warren [14], Theorem 1, Case A). As above, suppose the geometric random variables $g_{1, n-j+1}$ used in the update rule (4.1) from $\mathbf{G}^{p l}(n-1)$ to $\mathbf{G}^{p l}(n)$ have parameters $1-v_{1} v_{j}$ for $j=1, \ldots, n$. Then

$$
\begin{aligned}
P\left(\mathbf{G}^{p l}(n)=\left(y_{1}, \ldots, y_{n}\right) \mid \mathbf{G}^{p l}(n-1)=\left(x_{2}, \ldots, x_{n}\right)\right) & \\
& =\prod_{k=1}^{n}\left(1-v_{1} v_{k}\right)\left(v_{1} v_{k}\right)^{y_{k}-x_{k}} \operatorname{det}\left(w_{1,\left(1 /\left(v_{1} v_{k}\right)\right)_{k=1}^{n}(i j)}\left(y_{j}-x_{i}+j-i\right)\right)_{i, j=1}^{n}
\end{aligned}
$$

where $x_{1}:=0$ and $w_{1}(u)=1_{\{u \geq 0\}}$.

The proof uses the RSK correspondence; a more direct proof is given in the case with all parameters equal in [19] and with the geometric replaced by exponential data in [16].

We will iteratively apply these one-step updates and use the following lemma to find the probability mass function for $\mathbf{G}^{p l}(n)$ as a single determinant.

Lemma 4.2. Suppose that $p=\left(p_{1}, \ldots, p_{n}\right)$ and $p_{i}>0$ for $i=1, \ldots, n$. Let $\left(f_{i}\right)_{i=1}^{n}$ be a collection of functions from $\mathbb{Z}_{\geq 0} \rightarrow \mathbb{R}$ and $g: \mathbb{Z} \rightarrow \mathbb{R}$ with $g(u)=0$ for all $u<0$. Then

$$
\begin{aligned}
\sum_{x \in W_{\geq 0}^{n}} \operatorname{det}\left(D^{\left(1 / p_{2} \ldots 1 / p_{j}\right)} f_{i}\left(x_{j}+j-1\right)\right)_{i, j=1}^{n} \operatorname{det}\left(g_{1, p^{-1}}^{(i j)}\left(y_{j}-x_{i}+j-i\right)\right)_{i, j=1}^{n} & \\
& =\operatorname{det}\left(D^{\left(1 / p_{2} \ldots 1 / p_{j}\right)} \sum_{u \geq 0} f_{i}(u) g_{1}\left(y_{j}+j-1-u\right)\right)_{i, j=1}^{n} .
\end{aligned}
$$

Proof. We have $g(u)=0$ for all $u<0$ which means that $\left(J^{(p)} g\right)(z-\cdot)(u)=\left(I^{(1 / p)} g\right)(z-u)$. We apply Lemma 3.2 part (ii) with the functions $g_{j}(\cdot)=D^{\left(1 / p_{2} \ldots 1 / p_{j}\right)} g\left(y_{j}+j-1-\cdot\right)$ where $D^{\emptyset}=\mathrm{Id}$. We note that as $g$ is zero in a neighbourhood of infinity the condition on the growth of $f$ can be omitted.

\subsection{Proof of Theorem 1.2}

Proof. We prove by induction on $n$ that the probability mass function for $\mathbf{G}^{p l}(n)$ is given by the stationary distribution of PushASEP with a wall denoted by $\pi\left(x_{1}, \ldots, x_{n}\right)$ and given in Proposition 3.3. We note that the case $n=1$ holds. The proposed probability mass function for $\mathbf{G}^{p l}(n-1)$ is

$$
\pi\left(x_{2}, \ldots, x_{n}\right)=c_{n-1} \prod_{k=2}^{n} v_{k}^{x_{k}} \operatorname{det}\left(D_{x_{j}}^{\left(1 / v_{2} \ldots 1 / v_{j}\right)} \phi_{i}\left(x_{j}+j-2\right)\right)_{i, j=2}^{n}
$$

where $\phi_{i}(u)=v_{i}^{-(u+1)}-v_{i}^{u+1}$ for $i=2, \ldots, n$ and $c_{n-1}=\left(\prod_{2 \leq i<j \leq n}\left(v_{i}-v_{j}\right)\right)^{-1} \prod_{j=2}^{n} v_{j}^{n-1}$.

We define

$$
\hat{\pi}\left(x_{1}, \ldots, x_{n}\right)=c_{n-1} \prod_{k=2}^{n} v_{k}^{x_{k}} \operatorname{det}\left(D_{x_{j}}^{\left(1 / v_{2} \ldots 1 / v_{j}\right)} \hat{\phi}_{i}\left(x_{j}+j-2\right)\right)_{i, j=1}^{n}
$$

where $D^{\emptyset}=\operatorname{Id}, \hat{\phi}_{1}(u)=1_{\{u=-1\}}$ and $\hat{\phi}_{i}=\phi_{i}$ for each $i=2, \ldots, n$. We first show that for $\left(x_{1}, \ldots, x_{n}\right) \in W_{\geq 0}^{n}$,

$$
\hat{\pi}\left(x_{1}, \ldots, x_{n}\right)=1_{\left\{x_{1}=0\right\}} \pi\left(x_{2}, \ldots, x_{n}\right) .
$$

Consider a Laplace expansion of $\hat{\pi}$ where the summation is indexed by a permutation $\sigma$. If $\sigma(1)=1$ then the top-left entry in the matrix defining $\hat{\pi}$ is given by $\hat{\phi}_{1}\left(x_{1}-1\right)$ which equals 1 if $x_{1}=0$ and 0 otherwise. Therefore the terms in the Laplace expansion with $\sigma(1)=1$ will give the desired expression for $\hat{\pi}$ and we need to show the remaining terms in the Laplace expansion of $\hat{\pi}$ are zero. 
Let $\sigma(1)=j$ for some $2 \leq j \leq n$ and $\sigma(i)=1$ for some $2 \leq i \leq n$. For any $2 \leq j \leq n$, the $(1, j)$ entry in the matrix in (4.3) is only non-zero if $x_{j}=0$ by using the definition of $\hat{\phi}_{1}$. On the other hand, the $(i, 1)$ entry in the matrix in (4.3) is given by $\hat{\phi}_{i}\left(x_{1}-1\right)=v_{i}^{x_{1}}-v_{i}^{-x_{1}}=0$ if $x_{1}=0$. Therefore as $x_{1} \leq x_{j}$ all terms in the Laplace expansion of $\hat{\pi}$ with $\sigma(1) \neq 1$ are zero. This proves (4.4).

We use (4.3), (4.4) and the update rule in Lemma 4.1 to find the probability mass function for $\mathbf{G}^{p l}(n)$ as

$$
\begin{aligned}
c_{n-1} \sum_{\left(x_{2}, \ldots, x_{n}\right) \in W_{\geq 0}^{n-1}} \prod_{k=2}^{n} v_{k}^{x_{k}} \operatorname{det}\left(D_{x_{j}}^{\left(1 / v_{2} \ldots 1 / v_{j}\right)} \hat{\phi}_{i}\left(x_{j}+j-2\right)\right)_{i, j=1}^{n} \\
\cdot \prod_{k=1}^{n}\left(1-v_{1} v_{k}\right)\left(v_{1} v_{k}\right)^{y_{k}-x_{k}} \operatorname{det}\left(w_{1,\left(1 /\left(v_{1} v_{k}\right)\right)_{k=1}^{n}}^{(i j)}\left(y_{j}-x_{i}+j-i\right)\right)_{i, j=1}^{n}
\end{aligned}
$$

where $x_{1}:=0$. We use the identities:

$$
D^{(\alpha)} f(u)=\beta^{u} D^{(\alpha / \beta)}\left(f(u) \beta^{-u}\right), \quad I^{(\alpha)} f(u)=\beta^{u} I^{(\alpha / \beta)}\left(f(u) \beta^{-u}\right)
$$

to show that with $u=y_{j}-x_{i}+j-i$,

$$
\begin{aligned}
v_{1}^{u} I^{\left(1 /\left(v_{1} v_{j+1}\right), \ldots, 1 /\left(v_{1} v_{i}\right)\right)}\left(w_{1}(u)\right) & =I^{\left(1 / v_{j+1}, \ldots, 1 / v_{i}\right)}\left(w_{1}(u) v_{1}^{u}\right) \text { for } i>j \\
v_{1}^{u} D^{\left(1 /\left(v_{1} v_{i+1}\right), \ldots, 1 /\left(v_{1} v_{j}\right)\right)}\left(w_{1}(u)\right) & =D^{\left(1 / v_{i+1}, \ldots, 1 / v_{j}\right)}\left(w_{1}(u) v_{1}^{u}\right) \text { for } j>i .
\end{aligned}
$$

We use (4.6) and (4.7) in (4.5) to obtain the probability mass function for $\mathbf{G}^{p l}(n)$ as

$$
\begin{aligned}
c_{n-1} \sum_{\left(x_{2}, \ldots, x_{n}\right) \in W_{\geq 0}^{n-1}} \prod_{k=1}^{n} v_{k}^{y_{k}} \operatorname{det}\left(D_{x_{j}}^{\left(1 / v_{2} \ldots 1 / v_{j}\right)} \hat{\phi}_{i}\left(x_{j}+j-2\right)\right)_{i, j=1}^{n} \\
\cdot \prod_{k=1}^{n}\left(1-v_{1} v_{k}\right) \operatorname{det}\left(\hat{w}_{1, v^{-1}}^{(i j)}\left(y_{j}-x_{i}+j-i\right)\right)_{i, j=1}^{n}
\end{aligned}
$$

where $\hat{w}_{1}(u)=w_{1}(u) v_{1}^{u}$ and $\hat{w}_{1, v^{-1}}^{(i j)}$ is defined by (4.2). We apply Lemma 4.2 to show this equals

$$
c_{n-1} \prod_{k=1}^{n}\left(1-v_{1} v_{k}\right) v_{k}^{y_{k}} \operatorname{det}\left(D_{y_{j}}^{\left(1 / v_{2} \ldots 1 / v_{j}\right)} \sum_{u=0}^{y_{j}+j-1} \hat{\phi}_{i}(u-1) \hat{w}_{1}\left(y_{j}-u+j-1\right)\right)_{i, j=1}^{n} .
$$

In the case $i=1$, recall $\phi_{1}(x)=v_{1}^{(x+1)}-v_{1}^{-(x+1)}$ and observe that

$$
\sum_{u=0}^{y_{j}+j-1} \hat{\phi}_{1}(u-1) v_{1}^{y_{j}-u+j-1}=v_{1}^{y_{j}+j-1}=\frac{v_{1}}{1-v_{1}^{2}} D^{\left(1 / v_{1}\right)} \phi_{1}\left(y_{j}+j-1\right) .
$$

In the case $2 \leq i \leq n$ observe that

$$
\begin{aligned}
\sum_{u=0}^{y_{j}+j-1} \phi_{i}(u-1) v_{1}^{y_{j}-u+j-1} & =\frac{v_{i}^{y_{j}+j}}{v_{1}-v_{i}}-\frac{v_{1}^{y_{j}+j-1}}{1-v_{i} / v_{1}}+\frac{v_{1}^{y_{j}+j-1}}{1-1 /\left(v_{1} v_{i}\right)}-\frac{v_{i}^{-y_{j}-j+1}}{v_{1} v_{i}-1} \\
& =\frac{v_{i} v_{1}}{\left(1-v_{1} v_{i}\right)\left(v_{1}-v_{i}\right)} D^{\left(1 / v_{1}\right)} \phi_{i}\left(y_{j}+j-1\right)+C v_{1}^{y_{j}}
\end{aligned}
$$

where $C$ is independent of $y_{j}$. Using (4.9) and (4.10) in (4.8) and removing the terms $C v_{1}^{y_{j}}$ using row operations we find

$$
c_{n-1} \frac{v_{1}}{1-v_{1}^{2}} \prod_{j=2}^{n} \frac{v_{1} v_{j}}{\left(1-v_{1} v_{j}\right)\left(v_{1}-v_{j}\right)} \prod_{k=1}^{n}\left(1-v_{k} v_{1}\right) v_{k}^{y_{k}} \operatorname{det}\left(D_{y_{j}}^{\left(1 / v_{1} \ldots 1 / v_{j}\right)} \phi_{i}\left(y_{j}+j-1\right)\right)_{i, j=1}^{n} .
$$


The prefactor equals $c_{n}$ where $c_{n}=\prod_{1 \leq i<j \leq n} \frac{1}{\left(v_{i}-v_{j}\right)} \prod_{j=1}^{n} v_{j}^{n}$ and so we establish inductively that the probability mass function of $\mathbf{G}^{p l}(n)$ is given by

$$
\pi\left(y_{1}, \ldots, y_{n}\right)=c_{n} \prod_{k=1}^{n} v_{k}^{y_{k}} \operatorname{det}\left(D_{y_{j}}^{\left(1 / v_{1} \ldots 1 / v_{j}\right)} \phi_{i}\left(y_{j}+j-1\right)\right)_{i, j=1}^{n} .
$$

We recall that in Proposition 3.3, we deferred the proof of positivity of $\pi$ and the normalisation constant. This is now proven as we have identified $\pi$ as the probability mass function of $\mathbf{G}^{p l}(n)$. Moreover, Equation (4.11) and Proposition 3.3 proves Theorem 1.2 when $v_{1}, \ldots, v_{n}$ are distinct. The distribution of $\left(Y_{1}^{*}, \ldots, Y_{n}^{*}\right)$ is continuous in $\left(v_{1}, \ldots, v_{n}\right)$ on the set $(0,1)^{n}$ from Lemma 2.5 and the distribution of $(G(1, n), \ldots, G(1,1))$ is continuous in $\left(v_{1}, \ldots, v_{n}\right)$ on the same set as a finite number of operations of summation and maxima applied to geometric random variables. This completes the proof of Theorem 1.2 .

\subsection{The largest or rightmost particle}

Proposition 4.3. Let $F(\eta)=P\left(Y_{n}^{*} \leq \eta\right)=P(G(1,1) \leq \eta)$. For distinct $0<v_{1}, \ldots, v_{n}<$ 1 ,

$$
F(\eta)=\prod_{1 \leq i \leq j \leq n}\left(1-v_{i} v_{j}\right)\left(\prod_{j=1}^{n} v_{j}\right)^{\eta} \operatorname{Sp}_{\eta^{(n)}}\left(v_{1}, \ldots, v_{n}\right)
$$

where Sp denotes the symplectic Schur function from (2.2), and $\eta^{(n)}$ denotes an $n$ dimensional vector $(\eta, \ldots, \eta)$.

For point-to-line last passage percolation this was proven in [5] and related to earlier formulas for point-to-line last passage percolation in [2] and [6]. We could appeal to this and Theorem 1.1 to prove the same expression for the distribution function of $Y_{n}^{*}$. We now show that it follows quickly from Proposition 3.3.

Proof. From Proposition 3.3 we have

$$
F(\eta)=c_{n} \sum_{y_{1}, \ldots, y_{n} \leq \eta, y \in W_{\geq 0}^{n}} \prod_{k=1}^{n} v_{k}^{y_{k}} \operatorname{det}\left(D^{\left(1 / v_{1} \ldots 1 / v_{j}\right)} \phi_{i}\left(y_{j}+j-1\right)\right)_{i, j=1}^{n} .
$$

We perform the summation in $y_{n}$ from $y_{n-1}$ to $\infty$ which replaces the last column by $v_{n}^{\eta} D^{\left(1 / v_{1} \ldots 1 / v_{n-1}\right)} \phi_{i}(\eta+n-1)-v_{n}^{y_{n-1}-1} D^{\left(1 / v_{1} \ldots 1 / v_{n-1}\right)} \phi_{i}\left(y_{n-1}+n-2\right)$. The second term differs from the penultimate column by a factor of $v_{n}^{y_{n-1}-1} v_{n-1}^{-y_{n-1}}$ which is non-zero and independent of $i$. Therefore the second term can be removed from the last column by column operations. We now apply this procedure inductively in order $x_{n-1}, \ldots, x_{1}$ to obtain

$$
F(\eta)=c_{n}\left(\prod_{k=1}^{n} v_{k}\right)^{\eta} \operatorname{det}\left(D^{\left(1 / v_{2} \ldots 1 / v_{n-1}\right)} \phi_{i}(\eta+j-1)\right)_{i, j=1}^{n}
$$

where $D^{\emptyset}=$ Id. We relate this to a symplectic Schur function by using column operations. The entries in the first column are $\phi_{i}(\eta)=v_{i}^{-(\eta+1)}-v_{i}^{\eta+1}$. In the second column, the entries are $D^{\left(1 / v_{2}\right)} \phi_{i}(\eta+1)=\left(v_{i}^{-(\eta+2)}-v_{i}^{\eta+2}\right)-v_{2}^{-1}\left(v_{i}^{-(\eta+1)}-v_{i}^{\eta+1}\right)$ and the second bracketed term can be removed by column operations. This can be continued inductively and leads to

$$
\begin{aligned}
F(\eta) & =c_{n}(-1)^{n}\left(\prod_{k=1}^{n} v_{k}\right)^{\eta} \operatorname{det}\left(v_{i}^{\eta+j}-v_{i}^{-(\eta+j)}\right)_{i, j=1}^{n} \\
& =c_{n}\left(\prod_{k=1}^{n} v_{k}\right)^{\eta} \operatorname{Sp}_{\eta^{(n)}}\left(v_{1}, \ldots, v_{n}\right) \operatorname{det}\left(v_{i}^{j}-v_{i}^{-j}\right)_{i, j=1}^{n}
\end{aligned}
$$




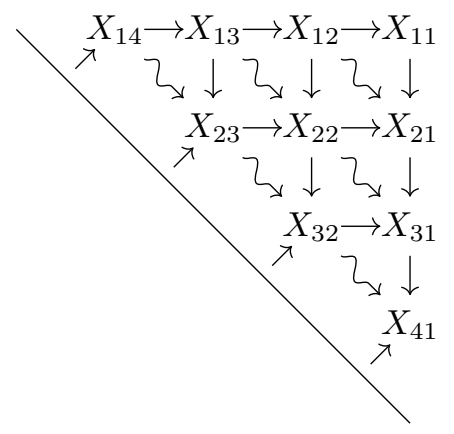

Figure 1: The interactions in the system $\left\{X_{i j}: i+j \leq n+1\right\}$.

The proof is now completed by using (2.5) to equate the normalisation constants.

\section{A dynamically reversible process}

We will suppose throughout that $0<v_{1}, \ldots, v_{n}<1$. Let $e_{i j}$ denote the vector taking value 1 in position $(i, j)$ and 0 otherwise. Let $S=\left\{(i, j): i, j \in \mathbb{Z}_{\geq 1}, i+j \leq n+1\right\}$ and

$$
\mathcal{X}=\left\{\left(x_{i j}\right)_{(i, j) \in S}: x_{i j} \in \mathbb{Z}_{\geq 0}, x_{i+1, j} \leq x_{i j} \text { and } x_{i, j+1} \leq x_{i j}\right\} .
$$

We define a continuous-time Markov process $\left(X_{i j}(t): i+j \leq n+1, t \geq 0\right)$ taking values in $\mathcal{X}$ by specifying its transition rates. For $x, x+e_{i j}+e_{i j-1}+\ldots+e_{i k} \in \mathcal{X},(i, j),(i, k) \in S$ and $k \leq j$ define

$$
q\left(x, x+e_{i j}+e_{i j-1}+\ldots+e_{i k}\right)=v_{n-j+1}\left(v_{n-j+1} v_{i-1}\right)^{-1_{\left\{x_{i j} \geq x_{i-1, j+1}\right\}}} 1_{\left\{x_{i j}=x_{i j-1}=\ldots=x_{i k}\right\}}
$$

with the notation that $x_{0, j}=\infty$ for $j=2, \ldots, n+1$. For $x, x-e_{i j}-e_{i+1 j}-\ldots-e_{l j} \in$ $\mathcal{X},(i, j),(l, j) \in S$ and $l \geq i$ define

$$
q\left(x, x-e_{i j}-e_{i+1 j}-\ldots-e_{l j}\right)=v_{n-j+1}^{-1}\left(v_{i-1} v_{n-j+1}\right)^{1_{\left\{x_{i j}>x_{i-1, j+1}\right\}}} 1_{\left\{x_{i j}=x_{i+1 j}=\ldots=x_{l j}\right\}} .
$$

All other transition rates are zero. The fact that $q\left(x, x^{\prime}\right) \neq 0$ only if $x, x^{\prime} \in \mathcal{X}$ corresponds to blocking interactions. This defines a multi-dimensional Markov chain with interactions shown in Figure 1, where the arrows in Figure 1 correspond to the following interactions.

(i) A push-block interaction denoted $A \rightarrow B$. If $A=B$ and $A$ jumps to the right by one then $B$ also jumps to the right by one (this may then cause further right jumps if $B \rightarrow C$ ). If $A=B$ and $B$ jumps left then this jump is suppressed.

(ii) A push-block interaction denoted $A \downarrow B$ where $A$ is at the base of the arrow and $B$ at the head of the arrow in Figure 1. If $A=B$ and $A$ jumps to the left by one then $B$ also jumps to the left by one (this may then causes further left jumps if $B \downarrow C$ ). If $A=B$ and $B$ jumps right then this jump is suppressed.

(iii) An interaction $A \leadsto B$ in which the rates of right and left jumps experienced by $B$ depend on its location relative to $A$. The particular form is given in (5.1) and (5.2) and is chosen such that $\left(X_{i j}(t): i+j \leq n+1, t \geq 0\right)$ is dynamically reversible, see part (ii) of Theorem 5.2.

(iv) An interaction with a wall in which all left jumps below zero are suppressed. This is depicted by the diagonal line on the left side of Figure 1.

To find the invariant measure of $X$ we use a result which has found applications in the queueing theory literature. 
Lemma 5.1 (Theorem 1.13, Kelly [20]). Let $X$ be a stationary Markov process with state space $E$ and transition rates $(q(j, k))_{j \neq k \in E}$. Suppose we can find positive sequences $(\hat{q}(j, k))_{j \neq k \in E}$ and $(\pi(j))_{j \in E}$ with $\sum_{j \in E} \pi(j)=1$ such that:

(i) $q(j)=\hat{q}(j)$ where $q(j)=\sum_{k \neq j} q(j, k)$ and $\hat{q}(j)=\sum_{k \neq j} \hat{q}(j, k)$.

(ii) $\pi(j) q(j, k)=\pi(k) \hat{q}(k, j)$.

Then $\pi$ is the invariant measure for $X$ and $\hat{q}$ are the transition rates of the time reversal of $X$ in stationarity.

The proof is straightforward: using (ii) then (i)

$$
\sum_{j \in E \backslash\{k\}} \pi(j) q(j, k)=\sum_{j \in E \backslash\{k\}} \pi(k) \hat{q}(k, j)=\pi(k) \hat{q}(k)=\pi(k) q(k) .
$$

Nonetheless this gives a convenient way of verifying an invariant measure if we can guess the transition rates of the time reversed process. In general, this is an intractable problem. However, in this case we can make the choice that the invariant measure is a field of point-to-line last passage percolation times and the reversed transition probabilities are given by reversing the direction of all interactions between particles in Figure 1 and changing the order of the parameters $\left(v_{1}, \ldots, v_{n}\right) \rightarrow\left(v_{n}, \ldots, v_{1}\right)$ (the interactions with the wall remain unchanged). This is motivated by the construction of [16].

More precisely, we define the reversed transition rates as follows. For $x, x+e_{i j}+$ $e_{i j-1}+\ldots+e_{i k} \in \mathcal{X}$ for $(i, j),(i, k) \in S$ and $k \leq i$ define

$$
\hat{q}\left(x+e_{i j}+e_{i j-1}+\ldots+e_{i k}, x\right)=v_{i}^{-1}\left(v_{n-k+2} v_{i}\right)^{1_{\left\{x_{i k} \geq x_{i+1, k-1}\right\}}} 1_{\left\{x_{i j}=x_{i j-1}=\ldots=x_{i k}\right\}}
$$

with the notation that $x_{j 0}=\infty$ for $j=2, \ldots, n+1$. For $x, x-e_{i j}-e_{i+1, j}-\ldots-e_{l j} \in \mathcal{X}$, $(i, j),(l, j) \in S$ and $l \geq i$, define

$$
\hat{q}\left(x-e_{i j}-e_{i+1, j}-\ldots-e_{l j}, x\right)=v_{l}\left(v_{n-j+2} v_{l}\right)^{-1_{\left\{x_{l j}>x_{l+1, j-1}\right\}}} 1_{\left\{x_{i j}=x_{i+1 j}=\ldots=x_{l j}\right\}} .
$$

Our proposed invariant measure is the probability mass function of $(G(i, j): i+j \leq$ $n+1)$. This has an explicit form:

$$
\pi(x)=\prod_{\{i+j<n+1\}}\left(1-v_{i} v_{n-j+1}\right)\left(v_{i} v_{n-j+1}\right)^{x_{i j}-\max \left(x_{i+1 j}, x_{i j+1}\right)} \prod_{i=1}^{n}\left(1-v_{i}^{2}\right) v_{i}^{2 x_{i n-i+1}}
$$

Theorem 5.2. Suppose that $0<v_{1}, \ldots, v_{n}<1$. Let $\left(X_{i j}^{\left(v_{1} \ldots v_{n}\right)}(t): i+j \leq n+1, t \geq 0\right)$ be the continuous-time Markov process with transition rates given by (5.1) and (5.2). This process has a unique invariant measure $\left(X_{i j}^{*}: i+j \leq n+1\right)$ which satisfies

$$
\left(X_{i j}^{*}: i+j \leq n+1\right) \stackrel{d}{=}(G(i, j): i+j \leq n+1)
$$

When run in stationarity,

$$
\left(X_{i j}^{\left(v_{1}, \ldots, v_{n}\right)}(t)\right)_{t \in \mathbb{R}, i+j \leq n+1} \stackrel{d}{=}\left(X_{j i}^{\left(v_{n}, \ldots, v_{1}\right)}(-t)\right)_{t \in \mathbb{R}, i+j \leq n+1}
$$

The process $\left(X_{i j}(t): i+j \leq n+1, t \geq 0\right)$ is irreducible, does not explode and has a unique invariant measure. The second statement is the statement that when run in stationarity $\left(X_{i j}(t): i+j \leq n+1, t \geq 0\right)$ is dynamically reversible. 
Proof. We will use Lemma 5.1. We first prove that for all $x, x^{\prime} \in \mathcal{X}$,

$$
\pi(x) q\left(x, x^{\prime}\right)=\pi\left(x^{\prime}\right) \hat{q}\left(x^{\prime}, x\right) .
$$

First consider the case when $x^{\prime}=x+e_{i j}+e_{i j-1}+\ldots+e_{i k}$. Both sides are zero unless $x_{i j}=x_{i j-1}=\ldots=x_{i k}$. When these equalities hold, $\max \left(x_{i p}, x_{i-1, p+1}\right)=x_{i-1, p+1}$ for each $p=j-1, \ldots, k$ and $\max \left(x_{i+1, p-1}, x_{i p}\right)=x_{i p}$ for each $p=j, \ldots, k+1$. Therefore when $x_{i j}=x_{i j-1}=\ldots=x_{i k}$,

$$
\pi(x)=\bar{\pi}_{1}\left(v_{i} v_{n-j+1}\right)^{x_{i j}}\left(v_{i-1} v_{n-j+1}\right)^{-x_{i j} 1_{\left\{x_{i j} \geq x_{i-1, j+1}\right\}}}\left(v_{i} v_{n-k+2}\right)^{-x_{i k} 1_{\left\{x_{i k} \geq x_{i+1, k-1}\right\}}}
$$

where $\bar{\pi}_{1}$ does not depend on $x_{i j}, x_{i j-1}, \ldots, x_{i k}$. In particular, with $x^{\prime}=x+e_{i j}+e_{i j-1}+$ $\ldots+e_{i k}$,

$$
\frac{\pi\left(x^{\prime}\right)}{\pi(x)}=v_{i} v_{n-j+1}\left(v_{i-1} v_{n-j+1}\right)^{-1_{\left\{x_{i j} \geq x_{i-1, j+1}\right\}}}\left(v_{i} v_{n-k+2}\right)^{-1_{\left\{x_{i k} \geq x_{i+1, k-1}\right\}} .}
$$

We compare to the ratio

$$
\frac{q\left(x, x^{\prime}\right)}{\hat{q}\left(x^{\prime}, x\right)}=v_{i} v_{n-j+1}\left(v_{i-1} v_{n-j+1}\right)^{-1_{\left\{x_{i j} \geq x_{i-1, j+1}\right\}}}\left(v_{i} v_{n-k+2}\right)^{-1_{\left\{x_{i k} \geq x_{i+1, k-1}\right\}} .}
$$

Combining the above two equations proves (5.3) in the case $x^{\prime}=x+e_{i j}+e_{i j-1}+\ldots+e_{i k}$. The second case is when $x^{\prime}=x-e_{i j}-e_{i+1, j}-\ldots-e_{l j}$ and proceeds in a similar manner. Both sides are zero unless $x_{i j}=x_{i+1 j}=\ldots=x_{l j}$. When these equalities hold, then $\max \left(x_{p-1 j}, x_{p, j-1}\right)=x_{p, j-1}$ for $p=l, \ldots, i+1$ and $\max \left(x_{p-1, j+1}, x_{p, j}\right)=x_{p j}$ for $p=l, \ldots, i+1$. Therefore

$$
\pi(x)=\bar{\pi}_{2}\left(v_{l} v_{n-j+1}\right)^{x_{l j}}\left(v_{l} v_{n-j+2}\right)^{-x_{l j} 1_{\left\{x_{l j}>x_{l+1, j-1}\right\}}}\left(v_{i-1} v_{n-j+1}\right)^{-x_{i j} 1_{\left\{x_{i j}>x_{i-1, j+1}\right\}}}
$$

where $\bar{\pi}_{2}$ does not depend on $x_{i j}, x_{i+1, j}, \ldots, x_{l j}$. Therefore letting $x^{\prime}=x-e_{i j}-e_{i+1, j}-$ $\ldots-e_{l j}$ we have

$$
\frac{\pi(x)}{\pi\left(x^{\prime}\right)}=\left(v_{l} v_{n-j+1}\right)\left(v_{l} v_{n-j+2}\right)^{-1_{\left\{x_{l j}>x_{l+1, j-1}\right\}}}\left(v_{i-1} v_{n-j+1}\right)^{-1_{\left\{x_{i j}>x_{i-1, j+1}\right\}}}
$$

and

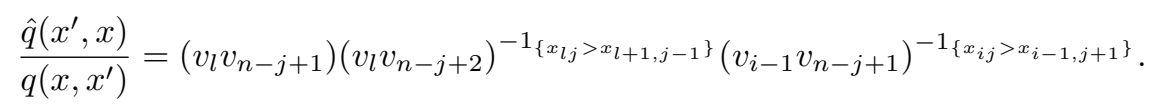

The two above equations prove (5.3) in the case when $x^{\prime}=x-e_{i j}-e_{i+1, j}-\ldots-e_{l j}$. Both sides of (5.3) are zero in all other cases and so we have proven (5.3).

We now show that for all $x \in \mathcal{X}$ we have $q(x)=\hat{q}(x)$. This follows from comparing,

$$
\begin{aligned}
& q(x)=v_{1}+ v_{1}^{-1} 1_{\left\{x_{1 n}>0\right\}}+\sum_{k=1}^{n-1} v_{n-k+1}+v_{n-k+1}^{-1} 1_{\left\{x_{1 k}>x_{1 k+1}\right\}} \\
&+\sum_{\{i \neq 1, i+j \leq n+1\}} v_{n-j+1}\left(v_{n-j+1} v_{i-1}\right)^{-1_{\left\{x_{i j} \geq x_{i-1, j+1}\right\}}} 1_{\left\{x_{i j}<x_{i-1, j}\right\}} \\
&+\sum_{\{i \neq 1, i+j<n+1\}} v_{n-j+1}^{-1}\left(v_{n-j+1} v_{i-1}\right)^{1_{\left\{x_{i j}>x_{i-1, j+1}\right\}}} 1_{\left\{x_{i j}>x_{i j+1}\right\}} \\
&+\sum_{\{i \neq 1, i+j=n+1\}} v_{n-j+1}^{-1}\left(v_{n-j+1} v_{i-1}\right)^{1_{\left\{x_{i j}>x_{i-1, j+1}\right\}}} 1_{\left\{x_{i j}>0\right\}}
\end{aligned}
$$


and

$$
\begin{aligned}
& \hat{q}(x)=\sum_{k=1}^{n-1} v_{k}+v_{k}^{-1} 1_{\left\{x_{k 1}>x_{k+1,1}\right\}}+v_{n}+v_{n}^{-1} 1_{\left\{x_{n 1}>0\right\}} \\
&+\sum_{\{i \neq 1, i+j \leq n+1\}} v_{i-1}\left(v_{i-1} v_{n-j+1}\right)^{-1_{\left\{x_{i-1, j+1} \geq x_{i j}\right\}}} 1_{\left\{x_{i-1, j+1}<x_{i-1, j}\right\}} \\
&+\sum_{\{i \neq 1, i+j<n+1\}} v_{i-1}^{-1}\left(v_{i-1} v_{n-j+1}\right)^{1_{\left\{x_{i-1, j+1}>x_{i j}\right\}}} 1_{\left\{x_{i-1, j+1}>x_{i, j+1}\right\}} \\
& \quad+\sum_{\{i \neq 1, i+j=n+1\}} v_{i-1}^{-1}\left(v_{i-1} v_{n-j+1}\right)^{1_{\left\{x_{i-1, j+1}>x_{i j}\right\}} 1_{\left\{x_{i-1, j+1}>0\right\}}} .
\end{aligned}
$$

One way to check that $q(x)=\hat{q}(x)$ is to check the equality first in the case when the inequalities $x_{i j}<x_{i-1, j}$ for each $i \neq 1, x_{i j}<x_{i j-1}$ for each $j \neq 1$ and $x_{i n-i+1}>0$ hold for each $i=1, \ldots, n$. This case can be seen directly from (5.4) and (5.5). We now consider the rates of jumps which are suppressed in each case when these inequalities no longer hold:

(i) If $x_{i, n-i+1}=0$ then both forwards and backwards in time a jump of rate $v_{i}^{-1}$ is suppressed by the wall.

(ii) If $x_{i j}=x_{i, j-1}$ then forwards in time the left jump of the $(i, j-1)$ particle is suppressed and the suppressed jump has rate $v_{n-j+2}^{-1}$ because $x_{i, j-1}=x_{i j} \leq x_{i-1, j}$. Backwards in time, the right jump of the $(i, j)$ particle is suppressed and the suppressed jump has rate $v_{n-j+2}^{-1}$ because $x_{i, j}=x_{i, j-1} \geq x_{i+1, j-1}$.

(iii) If $x_{i j}=x_{i-1 j}$ then forwards in time the right jump of the $(i, j)$ particle is suppressed and the suppressed jump has rate $v_{i-1}^{-1}$ because $x_{i j}=x_{i-1, j} \geq x_{i-1, j+1}$. Backwards in time, the left jump of the $(i-1, j)$ particle is suppressed and the suppressed jump has rate $v_{i-1}^{-1}$ because $x_{i-1 j}=x_{i j} \leq x_{i j-1}$.

Using Lemma 5.1, we have now established that $\pi$ is the invariant measure and $\hat{q}$ are the reversed transition rates in stationarity of $\left(X_{i j}(t): i+j \leq n+1, t \geq 0\right)$. The second statement in the Theorem follows from comparing $q$ and $\hat{q}$ and observing that they are identical after the swap $x_{i j} \rightarrow x_{j i}$ and $\left(v_{1}, \ldots, v_{n}\right) \rightarrow\left(v_{n}, \ldots, v_{1}\right)$.

We end by discussing two further properties of the the process $X$. These properties can both be proved by running the process $\left(X_{i j}(t): i+j \leq n+1, t \geq 0\right)$ in stationarity, forwards and backwards in time, and follow in exactly the same way as Section 5 of [16] as they depend on the structural properties of the $X$ array rather than the exact dynamics.

(i) The marginal distribution of any row $\left(X_{i, n-i+1}, \ldots, X_{i, 1}\right)$ run forwards in time is PushASEP with a wall with rate vector $\left(v_{i}, \ldots, v_{n}\right)$. The marginal distribution of any column $\left(X_{n-j+1, j}, \ldots, X_{1, j}\right)$ run backwards in time is PushASEP with a wall with rate vector $\left(v_{n-j+1}, \ldots, v_{1}\right)$

(ii) Let $Q_{t}^{n}$ denote the transition semigroup for PushASEP with a wall with $n$ particles. Let $P_{n-1 \rightarrow n}$ denote the transition kernel for the update of the Markov chain $\mathbf{G}^{\mathrm{pl}}$ defined in Section 4 from time $n-1$ to $n$. There is an intertwining between $Q_{t}^{n-1}$ and $Q_{t}^{n}$ with intertwining kernel given by $P_{n-1 \rightarrow n}$. In operator notation,

$$
Q_{t}^{n-1} P_{n-1 \rightarrow n}=P_{n-1 \rightarrow n} Q_{t}^{n} .
$$




\section{Push-block dynamics and Proposition 2.1}

The aim of this Section is to describe how Proposition 2.1 can be obtained by a construction of an interacting particle system with pushing and blocking interactions. This section is adapting the proof of Theorem 2.1 in [33] with a different intertwining (6.2) replacing Equation 3.3 from [33].

We follow the set-up and notation of [33]. For each $n \geq 1$, let $(\mathfrak{X}(t): t \geq 0)$ be a continuous-time Markov process $\mathfrak{X}(t)=\left(\mathfrak{X}_{i}^{j}(t)\right)_{1 \leq i \leq j \leq n}$ taking values in $\mathbb{K}_{n}=$ $\left\{\left(x_{i}^{j}\right)_{1 \leq i \leq j \leq n}\right.$ with $\left.x_{i}^{j+1} \leq x_{i}^{j} \leq x_{i+1}^{j+1}\right\}$. We use $x^{j}$ to denote the vector $x^{j}=\left(x_{1}^{j}, \ldots, x_{j}^{j}\right)$ and describe $\mathfrak{X}^{j}$ as the positions of the particles in the $j$-th level of $\mathfrak{X}$. Let $v_{i}>0$ for each $i \geq 1$.

The dynamics of $\mathfrak{X}$ is governed by $n(n+1)$ independent exponential clocks, where each particle in the $j$-th level has two independent exponential clocks with rates $v_{j}$ and $v_{j}^{-1}$ corresponding to its right and left jumps respectively. When the clock of a particle in the $j$-th level rings, that particle attempts to jump to the right or left but will experience a pushing and a blocking interaction which ensures that $\mathfrak{X}$ remains within $\mathbb{K}_{n}$. In summary, a particle at the $j$-th level pushes particles at levels $k>j$ and is blocked by particles at levels $k<j$. More precisely, suppose the right clock of $\mathfrak{X}_{i}^{j}$ rings.

(i) If $\mathfrak{X}_{i}^{j}=\mathfrak{X}_{i}^{j-1}$ then the right jump is suppressed.

(ii) If $\mathfrak{X}_{i}^{j}<\mathfrak{X}_{i}^{j-1}$ and $\mathfrak{X}_{i}^{j}=\mathfrak{X}_{i+1}^{j+1}$ then $\mathfrak{X}_{i}^{j}$ jumps right by one and pushes $\mathfrak{X}_{i+1}^{j+1}$ to the right by one. The right jump of $\mathfrak{X}_{i+1}^{j+1}$ may then cause further right jumps in the same way.

(iii) In all other cases $\mathfrak{X}_{i}^{j}$ jumps to the right by one and all other particles are unchanged.

If the left clock of $\mathfrak{X}_{i}^{j}$ rings then we have the same trichotomy of cases: (i) if $\mathfrak{X}_{i}^{j}=\mathfrak{X}_{i-1}^{j-1}$ then the left jump of $\mathfrak{X}_{i}^{j}$ is suppressed; (ii) if $\mathfrak{X}_{i}^{j}>\mathfrak{X}_{i-1}^{j-1}$ and $\mathfrak{X}_{i}^{j}=\mathfrak{X}_{i}^{j+1}$ then $\mathfrak{X}_{i}^{j}$ jumps to the left and pushes $\mathfrak{X}_{i}^{j+1}$ to the left by one which may then push further particles to the left; and (iii) in all other cases $\mathfrak{X}_{i}^{j}$ jumps to the left by one and all other particles are unchanged.

Let $n \geq 1$ and for $x \in \mathbb{K}_{n}$ let $w_{v}(x)=\prod_{i=1}^{n} v_{i}^{\left|x^{i}\right|-\left|x^{i-1}\right|}$ where $|x|=\sum_{j=1}^{d} x_{j}$ for $x \in \mathbb{R}^{d}$ and $\left|x^{0}\right|=0$. For any $z \in W^{n}$ we define $\mathbb{K}_{n}(z)=\left\{\left(x_{i}^{j}\right)_{1 \leq i \leq j \leq n} \in K_{n}: x^{n}=z\right\}$ and a probability measure on $\mathbb{K}_{n}(z)$ by $M_{z}(x)=w_{v}(x) / S_{z}(v)$ for all $x \in \mathbb{K}_{n}(z)$.

Proposition 6.1. Suppose $z \in W^{n}$ and that $(\mathfrak{X}(t): t \geq 0)$ has initial distribution $M_{z}(\cdot)$. Then $\left(\mathfrak{X}^{n}(t): t \geq 0\right)$ is a Markov process with conservative $Q$-matrix, given for $x \in W^{n}$ by

$$
Q\left(x, x \pm e_{i}\right)=\frac{S_{x \pm e_{i}}(v)}{S_{x}(v)} 1_{\left\{x \pm e_{i} \in W^{n}\right\}}, \text { for } i=1, \ldots, n
$$

with $Q(x, x)=-\sum_{i=1}^{n}\left(v_{i}^{-1}+v_{i}\right)$.

We prove this Proposition inductively in $n$ by analysing the two consecutive bottom layers of $\mathfrak{X}$ and include the statement that $Q$ is conservative as part of the induction argument. For $x \in W^{n}$ and $y \in W^{n+1}$ we will write $x \preceq y$ to mean that $y_{1} \leq x_{1} \leq y_{2} \leq \ldots x_{n} \leq$ $y_{n+1}$ and define $W^{n, n+1}=\left\{x \in W^{n}, y \in W^{n+1}: x \preceq y\right\}$. By the inductive hypothesis, the marginal distribution of the two consecutive bottom layers of $\mathfrak{X}$ is a continuous-time Markov process $(X(t), Y(t))_{t \geq 0}=\left(X_{1}(t), \ldots, X_{n}(t), Y_{1}(t), \ldots, Y_{n+1}(t)\right)_{t \geq 0}$ taking values in 
$W^{n, n+1}$ and with $Q$-matrix given by the off-diagonal entries: for $(x, y),\left(x^{\prime}, y^{\prime}\right) \in W^{n, n+1}$,

$$
\mathcal{A}\left((x, y),\left(x^{\prime}, y^{\prime}\right)\right)= \begin{cases}Q_{X}\left(x, x+e_{i}\right) & \text { if }\left(x^{\prime}, y^{\prime}\right)=\left(x+e_{i}, y\right) \text { and } x_{i}<y_{i+1}, \\ Q_{X}\left(x, x+e_{i}\right) & \text { if }\left(x^{\prime}, y^{\prime}\right)=\left(x+e_{i}, y+e_{i+1}\right) \text { and } x_{i}=y_{i+1}, \\ Q_{X}\left(x, x-e_{i}\right) & \text { if }\left(x^{\prime}, y^{\prime}\right)=\left(x-e_{i}, y\right) \text { and } x_{i}>y_{i}, \\ Q_{X}\left(x, x-e_{i}\right) & \text { if }\left(x^{\prime}, y^{\prime}\right)=\left(x-e_{i}, y-e_{i}\right) \text { and } x_{i}=y_{i}, \\ v_{n+1}^{ \pm} & \text {if }\left(x^{\prime}, y^{\prime}\right)=\left(x, y \pm e_{i}\right)\end{cases}
$$

with $Q_{X}$ given by the $Q$-matrix from Proposition 6.1. All other off-diagonal entries are zero and the diagonal entries $\mathcal{A}\left(\left(x^{\prime}, y^{\prime}\right),\left(x^{\prime}, y^{\prime}\right)\right)$ equal the negative of

$$
\sum_{i=1}^{n} v_{i}+\sum_{i=1}^{n} v_{i}^{-1}+\sum_{i=1}^{n} v_{n+1} 1_{\left\{y_{i}^{\prime}<x_{i}^{\prime}\right\}}+\sum_{i=1}^{n} v_{n+1}^{-1} 1_{\left\{y_{i+1}^{\prime}>x_{i}^{\prime}\right\}}+v_{n+1}+v_{n+1}^{-1}
$$

The inductive hypothesis that $Q_{X}$ is conservative means that $\mathcal{A}$ is conservative. We define the function

$$
m(x, y)=v_{n+1}^{|y|-|x|} \frac{S_{x}(v)}{S_{y}(v)}, \text { for }(x, y) \in W^{n, n+1}
$$

and an intertwining kernel given by $\Lambda: W^{n+1} \rightarrow W^{n, n+1}$

$$
\left.\Lambda\left(y,\left(x^{\prime}, y^{\prime}\right)\right)\right)=m\left(x^{\prime}, y^{\prime}\right) 1_{\left\{y=y^{\prime}\right\}} .
$$

The key step in proving Proposition 6.1 is to prove the intertwining $Q_{Y} \Lambda=\Lambda \mathcal{A}$ where $Q_{Y}$ is the desired $Q$-matrix from Proposition 6.1 with $n+1$ particles. This is equivalent to the statement that

$$
Q_{Y}\left(y, y^{\prime}\right)=\sum_{x \leq y} \frac{m(x, y)}{m\left(x^{\prime}, y^{\prime}\right)} \mathcal{A}\left((x, y),\left(x^{\prime}, y^{\prime}\right)\right), \text { for all } y, y^{\prime} \in W^{n}
$$

Once this is established it follows from general theory [28] that $Y$ is an autonomous Markov process with the desired $Q$-matrix and this $Q$-matrix is conservative; therefore Proposition 6.1 follows inductively. For a more detailed argument we refer to [33]: we are replacing Equation 3.3 from [33] with equation (6.2) and the rest of the argument is unchanged. It remains to show (6.2).

We first show (6.2) in the case $y^{\prime}=y$. The right hand side of (6.2) equals

$$
\sum_{x \preceq y} v_{n+1}^{\left|x^{\prime}\right|-|x|} \frac{S_{x}(v)}{S_{x^{\prime}}(v)} \mathcal{A}\left(\left(x, y^{\prime}\right),\left(x^{\prime}, y^{\prime}\right)\right)
$$

and $\mathcal{A}\left(\left(x, y^{\prime}\right),\left(x^{\prime}, y^{\prime}\right)\right)$ can be non-zero if $x=x^{\prime}$ or $x=x^{\prime} \pm e_{i}$. If $x=x^{\prime}$ then $\mathcal{A}\left(\left(x^{\prime}, y^{\prime}\right),\left(x^{\prime}, y^{\prime}\right)\right)$ equals the negative of (6.1). If $x=x^{\prime}-e_{i}$ then $\mathcal{A}\left(\left(x, y^{\prime}\right),\left(x^{\prime}, y^{\prime}\right)\right)=Q_{X}\left(x^{\prime}-e_{i}, x^{\prime}\right) 1_{\left\{y_{i}^{\prime}<x_{i}^{\prime}\right\}}$ and if $x=x^{\prime}+e_{i}$ then $\mathcal{A}\left(\left(x, y^{\prime}\right),\left(x^{\prime}, y^{\prime}\right)\right)=Q_{X}\left(x^{\prime}+e_{i}, x^{\prime}\right) 1_{\left\{y_{i+1}^{\prime}>x_{i}^{\prime}\right\}}$. Using these expressions we find that the right hand side of (6.2) is equal to

$$
\begin{aligned}
& -\left(\sum_{i=1}^{n} v_{i}+\sum_{i=1}^{n} v_{i}^{-1}+\sum_{i=1}^{n} v_{n+1} 1_{\left\{y_{i}^{\prime}<x_{i}^{\prime}\right\}}+\sum_{i=1}^{n} v_{n+1}^{-1} 1_{\left\{y_{i+1}^{\prime}>x_{i}^{\prime}\right\}}+v_{n+1}+v_{n+1}^{-1}\right) \\
& \quad+\sum_{i=1}^{n} v_{n+1} \frac{S_{x^{\prime}-e_{i}}(v)}{S_{x^{\prime}}(v)} \frac{S_{x^{\prime}}(v)}{S_{x^{\prime}-e_{i}}(v)} 1_{\left\{y_{i}^{\prime}<x_{i}^{\prime}\right\}}+\sum_{i=1}^{n} v_{n+1}^{-1} \frac{S_{x^{\prime}+e_{i}}(v)}{S_{x^{\prime}}(v)} \frac{S_{x^{\prime}}(v)}{S_{x^{\prime}+e_{i}}(v)} 1_{\left\{y_{i+1}^{\prime}>x_{i}^{\prime}\right\}}
\end{aligned}
$$

This equals $-\sum_{i=1}^{n+1} v_{i}-\sum_{i=1}^{n+1} v_{i}^{-1}=Q_{Y}\left(y^{\prime}, y^{\prime}\right)$ and proves (6.2) for $y^{\prime}=y$. 
Suppose that $y^{\prime}=y+e_{i}$ and consider two cases: depending on whether or not there was a pushing interaction. If $i=1$ or $i>1$ and $x_{i-1}^{\prime}<y_{i}^{\prime}$, the right hand side of (6.2) equals

$$
\frac{v_{n+1}^{\left|y^{\prime}-e_{i}\right|-\left|x^{\prime}\right|}}{v_{n+1}^{\left|y^{\prime}\right|-\left|x^{\prime}\right|}} \frac{S_{x^{\prime}}(v)}{S_{y^{\prime}-e_{i}}(v)} \frac{S_{y^{\prime}}(v)}{S_{x^{\prime}}(v)} v_{n+1}=Q_{Y}\left(y^{\prime}-e_{i}, y^{\prime}\right) .
$$

The second case is if $i>1$ and $x_{i-1}^{\prime}=y_{i}^{\prime}$, when the right hand side of (6.2) equals

$$
\frac{v_{n+1}^{\left|y^{\prime}-e_{i}\right|-\left|x^{\prime}-e_{i}\right|}}{v_{n+1}^{\left|y^{\prime}\right|-\left|x^{\prime}\right|}} \frac{S_{x^{\prime}-e_{i}}(v)}{S_{y^{\prime}-e_{i}}(v)} \frac{S_{y^{\prime}}(v)}{S_{x^{\prime}}(v)} \frac{S_{x^{\prime}}(v)}{S_{x^{\prime}-e_{i}}(v)}=Q_{Y}\left(y^{\prime}-e_{i}, y^{\prime}\right) .
$$

Finally suppose that $y^{\prime}=y-e_{i}$ and split again into two cases. If $i=n$ or $i<n$ and $y_{i}^{\prime}<x_{i}^{\prime}$ then the right hand side of (6.2) equals

$$
\frac{v_{n+1}^{\left|y^{\prime}+e_{i}\right|-\left|x^{\prime}\right|}}{v_{n+1}^{\left|y^{\prime}\right|-\left|x^{\prime}\right|}} \frac{S_{x^{\prime}}(v)}{S_{y^{\prime}+e_{i}}(v)} \frac{S_{y^{\prime}}(v)}{S_{x^{\prime}}(v)} v_{n+1}^{-1}=Q_{Y}\left(y^{\prime}+e_{i}, y^{\prime}\right)
$$

If $i<n$ and $y_{i}^{\prime}=x_{i}^{\prime}$ then the right hand side of (6.2) equals

$$
\frac{v_{n+1}^{\left|y^{\prime}+e_{i}\right|-\left|x^{\prime}+e_{i}\right|}}{v_{n+1}^{\left|y^{\prime}\right|-\left|x^{\prime}\right|}} \frac{S_{x^{\prime}+e_{i}}(v)}{S_{y^{\prime}+e_{i}}(v)} \frac{S_{y^{\prime}}(v)}{S_{x^{\prime}}(v)} \frac{S_{x^{\prime}}(v)}{S_{x^{\prime}+e_{i}}(v)}=Q_{Y}\left(y^{\prime}+e_{i}, y^{\prime}\right) .
$$

This completes the proof of (6.2) and as described above completes the proof of Proposition 6.1.

Proof of Proposition 2.1. We construct the process $\mathfrak{X}$ in Proposition 6.1 started from the origin with the rate of right and left jump rates on level $j$ given by $v_{n-j+1}$ and $v_{n-j+1}^{-1}$ respectively. Part (i) is an immediate consequence. Part (ii) follows from the fact that $\left(\mathfrak{X}_{n}^{n}(t)\right)_{t \geq 0}$ is the largest particle in two Markov processes $\left(\mathfrak{X}_{1}^{n}(t), \mathfrak{X}_{2}^{n}(t), \ldots, \mathfrak{X}_{n}^{n}(t)\right)_{t \geq 0}$ which has $Q$-matrix given in Proposition 6.1 and $\left(\mathfrak{X}_{1}^{1}(t), \mathfrak{X}_{2}^{2}(t), \ldots, \mathfrak{X}_{n}^{n}(t)\right)_{t \geq 0}$ which is PushASEP (without a wall) where the $i$-th particle has right jump rate $v_{n-i+1}$ and left jump rate $v_{n-i+1}$. The top particle of PushASEP (without a wall) is equal in distribution as a process to the right hand side of Proposition 2.1 by the argument used to prove equation (2.8).

\section{References}

[1] J. Baik, P.A. Deift, and K. Johansson, On the distribution of the length of the longest increasing subsequence of random permutations, J. Amer. Math. Soc. 12 (1999), 1119-1178. MR1682248

[2] Jinho Baik and Eric M. Rains, Algebraic aspects of increasing subsequences, Duke Math. J. 109 (2001), no. 1, 1-65. MR-1844203

[3] Yu. M. Baryshnikov, GUEs and queues, Probab. Theory Related Fields 119 (2001), no. 2, 256-274. MR-1818248

[4] Philippe Biane, Philippe Bougerol, and Neil O'Connell, Littelmann paths and Brownian paths, Duke Math. J. 130 (2005), no. 1, 127-167. MR-2176549

[5] Elia Bisi and Nikos Zygouras, Transition between characters of classical groups, decomposition of Gelfand-Tsetlin patterns and last passage percolation, arXiv:1905.09756.

[6] Elia Bisi and Nikos Zygouras, Point-to-line polymers and orthogonal Whittaker functions, Transactions of the American Mathematical Society 371 (2017), 8339-8379. MR-3955549

[7] Alexei Borodin and Ivan Corwin, Macdonald processes, Probability Theory and Related Fields 158 (2014), no. 1, 225-400. MR-3152785 
[8] Alexei Borodin and Patrik Ferrari, Large time asymptotics of growth models on space-like paths I: PushASEP, Electron. J. Probab. 13 (2008), 1380-1418. MR-2438811

[9] Alexei Borodin, Patrik Ferrari, Michael Prähofer, Tomohiro Sasamoto, and Jon Warren, Maximum of Dyson Brownian motion and non-colliding systems with a boundary, Electron. Commun. Probab. 14 (2009), 486-494. MR-2559098

[10] Alexei Borodin, Patrik L. Ferrari, Michael Prähofer, and Tomohiro Sasamoto, Fluctuation properties of the TASEP with periodic initial configuration, J Stat Phys 129 (2007), 1055-1080. MR-2363389

[11] Peter Clifford and Aidan Sudbury, A sample path proof of the duality for stochastically monotone Markov processes, Ann. Probab. 13 (1985), no. 2, 558-565. MR-0781422

[12] Ivan Corwin, Neil O'Connell, Timo Seppäläinen, and Nikos Zygouras, Tropical combinatorics and Whittaker functions, Duke Math. J. 163 (2014), no. 3, 513-563. MR-3165422

[13] J. Theodore Cox and Uwe Rösler, A duality relation for entrance and exit laws for Markov processes, Stochastic Processes and their Applications 16 (1984), no. 2, 141-156. MR0724061

[14] A. B. Dieker and J. Warren, Determinantal transition kernels for some interacting particles on the line, Ann. Inst. H. Poincaré Probab. Statist. 44 (2008), no. 6, 1162-1172. MR-2469339

[15] P. L. Ferrari, Polynuclear growth on a flat substrate and edge scaling of GOE eigenvalues, Comm. Math. Phys. 252 (2004), 77-109. MR-2103905

[16] Will FitzGerald and Jon Warren, Point-to-line last passage percolation and the invariant measure of a system of reflecting Brownian motions, Probability Theory and Related Fields 178 (2020), no. 1, 121-171. MR-4146536

[17] W. Fulton and J. Harris, Representation theory. a first course, volume 129 of graduate texts in mathematics, Springer-Verlag, 1991. MR-1153249

[18] K. Johansson, Shape fluctuations and random matrices, Comm. Math. Phys. 209 (2000), 437-476. MR-1737991

[19] Kurt Johansson, A multi-dimensional Markov chain and the Meixner ensemble, Ark. Mat. 48 (2010), no. 1, 79-95. MR-2594587

[20] F. P. Kelly, Reversibility and stochastic networks, Cambridge University Press, USA, 2011. MR-2828834

[21] Wolfgang König, Orthogonal polynomial ensembles in probability theory, Probab. Surveys 2 (2005), 385-447. MR-2203677

[22] Wolfgang König, Neil O’Connell, and Sébastien Roch, Non-colliding random walks, tandem queues, and discrete orthogonal polynomial ensembles, Electron. J. Probab. 7 (2002), 24 pp. MR-1887625

[23] Cédric Lecouvey, Emmanuel Lesigne, and Marc Peigné, Random walks in Weyl chambers and crystals, Proceedings of the London Mathematical Society 104 (2012), no. 2, 323-358. MR-2880243

[24] Gia Bao Nguyen and Daniel Remenik, Non-intersecting Brownian bridges and the Laguerre Orthogonal Ensemble, Ann. Inst. H. Poincaré Probab. Statist. 53 (2017), no. 4, 2005-2029. MR-3729645

[25] Neil O'Connell, Conditioned random walks and the RSK correspondence, Journal of Physics A: Mathematical and General 36 (2003), no. 12, 3049-3066. MR-1986407

[26] Neil O'Connell, Directed polymers and the quantum Toda lattice, Ann. Probab. 40 (2012), no. 2, 437-458. MR-2952082

[27] M. Prähofer and H. Spohn, Scale invariance of the PNG droplet and the Airy process, J. Stat. Phys. 108 (2002), 1071-1106. MR-1933446

[28] L. C. G. Rogers and J. W. Pitman, Markov functions, Ann. Probab. 9 (1981), no. 4, 573-582. MR-0624684

[29] T. Sasamoto, Spatial correlations of the 1d KPZ surface on a flat substrate, Journal of Physics A: Math. and Gen. 38 (2005), no. 33, L549. MR-2165697

[30] Gunter M. Schütz, Exact solution of the master equation for the asymmetric exclusion process, J. Statist. Phys. 88 (1997), no. 1-2, 427-445. MR-1468391 
[31] Craig A. Tracy and Harold Widom, Asymptotics in ASEP with step initial condition, Communications in Mathematical Physics 290 (2009), no. 1, 129-154. MR-2520510

[32] Jon Warren, Dyson's Brownian motions, intertwining and interlacing, Electron. J. Probab. 12 (2007), 573-590. MR-2299928

[33] Jon Warren and Peter Windridge, Some examples of dynamics for Gelfand-Tsetlin patterns, Electron. J. Probab. 14 (2009), 1745-1769. MR-2535012

Acknowledgments. I am very grateful to Jon Warren for helpful and stimulating discussions and to Neil O'Connell for suggesting the approach in Section 2.1. 